\title{
Buried AGNs in Advanced Mergers: Mid-infrared Color Selection as a Dual AGN Candidate Finder
}

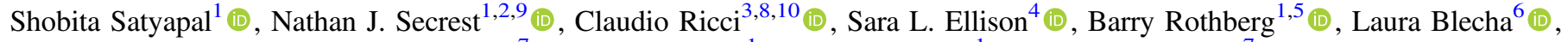 \\ Anca Constantin ${ }^{7}$ (i), Mario Gliozzi ${ }^{1}{ }^{1}$, , Paul McNulty ${ }^{1}$, and Jason Ferguson ${ }^{7}$ \\ ${ }^{1}$ George Mason University, Department of Physics \& Astronomy, MS 3F3, 4400 University Dr., Fairfax, VA 22030, USA \\ ${ }^{2}$ National Academy of Sciences NRC Research Associate, 2101 Constitution Ave. NW, Washington, DC 20418, USA \\ ${ }^{3}$ Instituto de Astrofsica, Facultad de Física, Pontificia Universidad Católica de Chile, Casilla 306, Santiago 22, Chile \\ ${ }^{4}$ Department of Physics and Astronomy, University of Victoria, Victoria, BC V8P 1A1, Canada \\ ${ }^{5}$ LBT Observatory, University of Arizona, 933 N. Cherry Ave., Tuscan, AZ 85721, USA \\ ${ }^{6}$ Astronomy Department, University of Maryland, College Park, MD 20742, USA \\ ${ }^{7}$ Department of Physics and Astronomy, James Madison University, Harrisonburg, VA 22807, USA \\ ${ }^{8}$ Chinese Academy of Sciences South America Center for Astronomy and China-Chile Joint Center for Astronomy, \\ Camino El Observatorio 1515, Las Condes, Santiago, Chile \\ Received 2017 March 15; revised 2017 August 12; accepted 2017 August 20; published 2017 October 23
}

\begin{abstract}
A direct consequence of hierarchical galaxy formation is the existence of dual supermassive black holes, which may be preferentially triggered as active galactic nuclei (AGNs) during galaxy mergers. Despite decades of searching, however, dual AGNs are extremely rare, and most have been discovered serendipitously. Using the all-sky WISE survey, we identified a population of over 100 morphologically identified interacting galaxies or mergers that display red mid-infrared colors often associated in extragalactic sources with powerful AGNs. The vast majority of these advanced mergers are optically classified as star-forming galaxies, which suggests that they may represent an obscured population of AGNs that cannot be found through optical studies. In this work, we present Chandra/ACIS observations and near-infrared spectra with the Large Binocular Telescope of six advanced mergers with projected pair separations less than $\sim 10 \mathrm{kpc}$. The combined X-ray, near-infrared, and mid-infrared properties of these mergers provide confirmation that four out of the six mergers host at least one AGN, with four of the mergers possibly hosting dual AGNs with projected separations less than $\sim 10 \mathrm{kpc}$, despite showing no firm evidence for AGNs based on optical spectroscopic studies. Our results demonstrate that (1) optical studies miss a significant fraction of single and dual AGNs in advanced mergers, and (2) mid-infrared pre-selection is extremely effective in identifying dual AGN candidates in late-stage mergers. Our multi-wavelength observations suggest that the buried AGNs in these mergers are highly absorbed, with intrinsic column densities in excess of $\sim N_{\mathrm{H}}>10^{24} \mathrm{~cm}^{-2}$, consistent with hydrodynamic simulations.
\end{abstract}

Key words: black hole physics - galaxies: active - galaxies: evolution - galaxies: interactions - infrared: galaxies $\mathrm{X}$-rays: galaxies

\section{Introduction}

According to the current cold dark matter cosmological paradigm, galaxy interactions are an integral part of a galaxy's cosmic history and play a critical role in its evolution. Theory predicts that these interactions funnel gas toward the central regions of galaxies (Mihos \& Hernquist 1996), potentially triggering gas accretion onto the central supermassive black hole (SMBH) and causing it to shine brightly as an active galactic nucleus (AGN). Although a minority of AGNs by number appear to be hosted in ongoing mergers at both low $(z<1)$ (e.g., Cisternas et al. 2011; Villforth et al. 2014) and high $(z \sim 2)$ redshift (e.g., Schawinski et al. 2011; Kocevski et al. 2012; Fan et al. 2014; Mechtley et al. 2016; Villforth et al. 2016), observations and semi-empirical modeling suggest that merger-triggered AGNs may dominate SMBH growth, especially at the highest luminosities (e.g., Treister et al. 2012; Hopkins et al. 2014). Also, as the vast majority of galaxies are thought to contain SMBHs, a direct consequence of the hierarchical model of galaxy formation should be the

\footnotetext{
${ }^{9}$ Resident at Naval Research Laboratory, 4555 Overlook Ave SW, Washington, DC 20375, USA.

${ }^{10}$ Kavli Institute for Astronomy and Astrophysics, Peking University, Beijing 100871, China.
}

existence of gravitationally bound binary AGNs, the spatially resolvable precursors of which would be dual AGNs with separations of a few kiloparsecs. Detections of such objects provide unambiguous confirmation of active SMBH growth during late-stage mergers, and the simultaneous fueling of both AGNs indicates that these are very efficient environments for triggering SMBH accretion. Since accretion onto both SMBHs occurs in late stage mergers when the accretion rate is expected to be the highest (Van Wassenhove et al. 2012; Blecha et al. 2013), dual AGNs likely coincide with the period of the most rapid black hole growth and therefore represent a key stage in the evolution of galaxies, which contributes significantly to the SMBH accretion history of the universe. Moreover, dual AGNs are the likely precursors of SMBH binaries and mergers, which will be the loudest gravitational wave sirens in the universe (Merritt \& Milosavljević 2005), the detection of which marks an exciting new era in astrophysics, as demonstrated by the lower-mass binary black holes detected by LIGO (Abbott et al. 2016). Future gravitational wave studies of black hole binaries and mergers in the SMBH range with Pulsar Timing Arrays (PTAs) and Space Laser Interferometry will enable precise measurements of black hole masses and spins, providing important constraints on the formation, accretion, and merger history of SMBHs. While dual AGNs are not gravitationally 
bound, and only a fraction of dual AGNs will coalesce within a Hubble time, as upper limits of the stochastic Gravitational Wave Background suggest (Shannon et al. 2015; Arzoumanian et al. 2016; Lentati et al. 2016; Verbiest et al. 2016), the frequency, mass distribution, and localization of this population will provide important insight into the properties, spatial distribution, and expected frequency and duration of the observationally less accessible binary phase. Therefore, a firm understanding of the frequency and properties of dual AGNs is crucial for our overall understanding of $\mathrm{SMBH}$ and galaxy evolution.

\subsection{The Rarity of Dual AGNs}

Despite decades of searching, and strong theoretical reasons why they should exist, dual AGNs are extremely rare. Indeed, only $0.1 \%$ of quasars are found in pairs with projected separations of tens to hundreds of kiloparsecs (e.g., Foreman et al. 2009; Hennawi et al. 2010), and until recently only a handful of confirmed dual AGNs with projected separations less than $10 \mathrm{kpc}$ were known in the universe (e.g., NGC 6240: Komossa et al. 2003; Mrk 463: Bianchi et al. 2008; Arp 299: Ballo et al. 2004; 3C 75: Owen et al. 1985; Radio Galaxy 0402+379: Rodriguez et al. 2006; Was49: Moran et al. 1992; Secrest et al. 2017), all of which were discovered serendipitously. In the past few years, with the advent of large-scale optical spectroscopic surveys, more systematic surveys of dual AGNs have been possible. In particular, $1 \%$ of low redshift AGNs identified by the Sloan Digital Sky Survey (SDSS) display double-peaked [O III] $\lambda 5007$ emission (e.g., Wang et al. 2009; Liu et al. 2010; Smith et al. 2010), a possible signature of SMBHs in orbital motion on kiloparsec scales. A few of these sources have been confirmed to be dual AGNs with separations of less than $10 \mathrm{kpc}$ by follow-up high spatial resolution imaging observations (e.g., Comerford et al. 2011, 2013, 2015; Fu et al. 2012; Liu et al. 2013; McGurk et al. 2015). While this is a promising avenue of investigation, only a small fraction $(\approx 2 \%)$ of the doubled peaked emitters have been confirmed to be dual AGNs (e.g., Shen et al. 2011; Comerford et al. 2012; Fu et al. 2012; Müller-Sánchez et al. 2015). A significant impediment to this technique is the ambiguity of the optical signatures. Double-peaked emission line profiles can also be produced by rotating disks or bi-conical outflows of the narrow line region gas surrounding single AGNs (e.g., Smith et al. 2012; Gabányi et al. 2014), a likely explanation for a large fraction of the candidates (e.g., Fu et al. 2012). Moreover, hydrodynamic simulations predict double peak narrow lines induced by the motion of dual AGNs for only a small fraction of the merger timescale (Blecha et al. 2013).

An even bigger concern is that dual AGNs may be optically obscured for a large fraction of the time when they are active, as expected during late stage mergers, where dual AGNs are expected to be found. Indeed, mid-infrared color selection with the Wide-field Infrared Sky Explorer Survey (WISE; Wright et al. 2010) has been demonstrated to yield a significantly higher AGN detection rate than optical studies in the most advanced mergers, which are known to be dusty (Satyapal et al. 2014). This result is consistent with the findings that the host morphologies of heavily obscured AGNs show a higher fraction of merger signatures compared with unobscured AGNs (Kocevski et al. 2015; Ricci et al. 2017). This is further suggested by the recent study by Fan et al. (2016), which showed that the host morphologies of hot dust-obscured galaxies (Hot DOGs) relative to a control sample are significantly disturbed compared with a
UV/optical-selected, unobscured AGN sample, consistent with a scenario in which the most luminous obscured AGN population is merger-driven, in contrast to the unobscured AGN population. Given the scarcity of observations, and the lack of extensive investigations that are carried out at wavelengths less sensitive to extinction, it is not yet possible to determine the true frequency of dual AGNs and to uncover the AGN and host galaxy properties during a key stage in the co-evolution of SMBHs and galaxies.

\subsection{The Power of WISE in Identifying Dual AGN Candidates}

Given the rarity of dual AGNs, a systematic approach to finding them is essential to increase the number of confirmed cases. The all-sky survey carried out by WISE has opened up a new window in the search for optically hidden AGNs in a large number of galaxies. This is because hot dust surrounding AGNs produces a strong mid-infrared continuum and infrared spectral energy distribution (SED) that is clearly distinguishable from star-forming galaxies in both obscured and unobscured AGNs (e.g., Stern et al. 2012). In particular, at low redshift, the $W 1(3.4 \mu \mathrm{m})-W 2(4.6 \mu \mathrm{m})$ color of galaxies dominated by AGNs is considerably redder than that of inactive galaxies (see Figure 1 in Stern et al. 2012; Assef et al. 2013). We can therefore use the WISE survey to identify a sample of AGN candidates drawn from a large sample of nearby interacting galaxies for follow-up investigation. Such a technique specifically targets the optically obscured dual AGN population and is complementary to current optical spectroscopic investigations.

In this paper, we use mid-infrared color selection with WISE as a preselection strategy for finding dual AGNs missed by optical studies in a large sample of advanced mergers. We present our first follow-up Chandra observations of our sample, together with ground-based near-infrared spectra obtained with the Large Binocular Telescope (LBT). In Section 2, we describe our sample selection strategy followed by a description of our X-ray and nearinfrared ground-based observations and data analysis in Section 3. In Section 4, we discuss our results. In Section 5 we explore the nature of the nuclear source and describe the multiwavelength diagnostics used in this work to ascertain the presence of an AGN in our sample. In Section 6, we describe the details of our observational diagnostics in each merger in the sample, followed by a discussion of our results in Section 7. We summarize our findings in Section 8.

All object coordinates (J2000) and redshifts used in this paper are taken from the SDSS tenth data release (DR10). ${ }^{11}$ We adopt $H_{0}=70 \mathrm{~km} \mathrm{~s}^{-1} \mathrm{Mpc}^{-1}, \Omega_{\mathrm{M}}=0.3$, and $\Omega_{\Lambda}=0.7$ for distance calculations. Luminosity and angular size distances were calculated using Ned Wright's cosmology calculator (Wright 2006). ${ }^{12}$

\section{Sample Selection}

Using the Galaxy Zoo project (Lintott et al. 2008), ${ }^{13}$ we assembled a large sample of interacting galaxies from the SDSS DR7. Here, Galaxy Zoo users were asked to identify morphological signs of interactions by selecting a "merger" button. Of all the galaxies in the SDSS database, 687,827 had Galaxy Zoo classifications available. We used the weightedmerger-vote-fraction, $f_{m}$, to explore the interaction status of the

\footnotetext{
11 https://www.sdss3.org/dr10

12 http://www.astro.ucla.edu/ wright/CosmoCalc.html

13 http://www.galaxyzoo.org
} 
sample. This parameter varies from 0 to 1 , where 0 represents clearly isolated galaxies and a value of 1 represents a definitive merger (Darg et al. 2010). We searched the All-WISE release of the WISE catalog, ${ }^{14}$ where a galaxy is considered matched if the positions agree to within 6 arcsec, for galaxies with $f_{m}>0.7$ and WISE detections in the first 2 bands with signal to noise greater than $5 \sigma$. There are 1372 galaxies that meet this criterion.

Of the assembled sample of merger candidates, we searched for objects that had mid-infrared signatures suggestive of AGNs. There are several WISE color diagnostics that have been used extensively in the literature to select AGNs (e.g., Donley et al. 2007; Jarrett et al. 2011; Mateos et al. 2012; Stern et al. 2012). The efficacy of these color cuts have been shown to be highly AGN luminosity-dependent, often missing a significant fraction of independently confirmed well-studied bona fide AGNs, even at moderate luminosities. For example, Mateos et al. (2012) adopted a stringent three-band color cut using the first 3 WISE bands that reliably identifies $97.1 \%$ of the luminous $\left(L_{2-10 \mathrm{keV}}>10^{44} \mathrm{erg} \mathrm{s}^{-1}\right)$ AGNs in their ultra hard-X-rayselected sample, but found that at luminosities $L_{2-10 \mathrm{keV}}<10^{44}$ $\mathrm{erg} \mathrm{s}^{-1}$, only $39.1 \%$ of the type 2 AGNs are identified (See also Section 4.4 in Secrest et al. 2015a). Similarly, among the Swift/ BAT AGNs from the 70 month catalog (Baumgartner et al. 2013), which are the most complete sample of hard X-ray (14-195 keV) selected AGNs in the local universe, Ichikawa et al. (2017) showed that only $\sim 10 \%$ of the AGNs in the luminosity range of $10^{42} \mathrm{erg} \mathrm{s}^{-1}<L_{14-195 \mathrm{keV}}<$ $10^{43} \mathrm{erg} \mathrm{s}^{-1}$ are identified by the widely used color cut of $W 1-W 2>0.8$ from Stern et al. (2012). Since our goal is to use mid-infrared color selection as a pre-selection strategy to identify AGN candidates for follow-up studies, we adopt in this work a more inclusive color cut of $W 1-W 2>0.5$ to increase the original sample size from which our candidates are selected. We chose this color cut because the galaxies in our sample are all nearby, and at redshifts below 0.2 , even the most extreme star-forming templates from Assef et al. (2013), have $W 1-W 2$ color well below 0.5 . We demonstrated in our previous study of interacting galaxies (Satyapal et al. 2014) that both $W 1-W 2>0.5$ and $W 1-W 2>0.8$ color cuts produce qualitatively similar results. We note that hydrodynamic merger simulations that include radiative transfer predict that the $W 1-W 2$ color rises above 0.5 just before the stage of peak black hole growth, where star formation rates (SFRs) are high and where dual AGNs are expected to be found (L. Blecha et al. 2017, in preparation; see also Snyder et al. 2013; Roebuck et al. 2016, for simulations of the mid-infrared spectral energy distribution with varying contributions of $\mathrm{AGN}$ and star formation activity), and that a color cut of $W 1-W 2>0.5$ is actually more effective at finding dual AGN candidates than is the $W 1-W 2>0.8$ color cut (L. Blecha et al. 2017, in preparation). We emphasize that the adopted color cut of $W 1-W 2>0.5$ is optimized to provide the most inclusive sample of merger AGN candidates. The follow-up multiwavelength observations presented in this work are required to provide confirmation for the existence of an AGN. We note that the galaxy nuclei in the assembled interacting sample are generally not resolved by WISE. The WISE color selection therefore ensures a high probability that at least one of the galaxies has an AGN. Of the 1372 galaxies with $f_{m}>0.7$, we

\footnotetext{
${ }^{14}$ http://wise2.ipac.caltech.edu/docs/release/allwise/
}

identified 112 galaxies that meet our adopted WISE color cut, 90 of which had two clear stellar nuclear concentrations identifiable in SDSS with spatial separations resolvable by Chandra $\left(>1^{\prime \prime}\right)$.

In our pilot Cycle 15 Chandra study (PID: 15700338; Satyapal), we obtained follow-up X-ray observations of six targets with the brightest predicted X-ray fluxes based on their mid-infrared flux in the assembled WISE sample with two clear nuclei spatially resolvable by Chandra and pair projected separations less than $10 \mathrm{kpc}$ that had not been previously observed by Chandra. We chose this pair separation cutoff since dual AGNs at these pair separations are extremely rare, and this pairing phase allows us to probe the stage of most active black hole growth and the only spatially observationally accessible precursors to the true binary AGN phase (Van Wassenhove et al. 2012; Blecha et al. 2013). Our working definition of a dual AGN in this paper corresponds to a merger with two confirmed nuclear AGNs with pair separations of less than $10 \mathrm{kpc}$. In Figure 1, we show three color SDSS images of our targets. As can be seen from the SDSS images, all targets are strongly disturbed systems, suggesting they are advanced mergers. In Table 1, we list the basic properties of the sources. Redshifts, stellar masses, and emission line fluxes for the galaxies in our sample were taken from the Max Planck Institut für Astrophysik/ Johns Hopkins University (MPA/JHU) collaboration. ${ }^{15}$ SDSS spectra are available for both nuclei in only SDSS J0122+0100 and SDSS J1045+3519 (SDSS fiber locations are displayed in Figure 1). For SDSS J1221+1137, there is an SDSS spectra for Gal 1 but no SDSS spectrum for Gal 2. However, there is another SDSS spectrum of a northern source that is not coincident with either possible Chandra source. As can be seen from the SDSS images, the targets have highly disturbed morphologies, making it difficult to precisely constrain the location of the galaxy nuclei and obtain meaningful estimates of their stellar masses and mass ratios. The optical spectral class of each target was determined using the BPT line ratio diagnostics of Baldwin et al. (1981), following the classification schemes of Kewley et al. (2001) for AGNs and Kauffmann et al. (2003) for composites. None of the six mergers are identified optically as dual AGNs, as can be seen from the bottom panels in Figure 1. Five out of the six mergers had detections in all four IRAS bands. Their $8-1000 \mu \mathrm{m} \mathrm{IR}$ luminosities, calculated using the prescription from Sanders \& Mirabel (1996), are all above or approximately equal to $10^{11} L_{\odot}$, placing them in the class of luminous infrared galaxies (LIRGs). Note that the IRAS beam encompasses both galaxies for these targets.

\section{Observations and Data Reduction}

\subsection{Chandra ACIS Imaging Observations}

The Chandra observations of the six mergers were carried out with ACIS-S between 2014 June and 2015 February with the source at the aim point of the S3 chip. The exposure times ranged from 3 to $16 \mathrm{ks}$ and were based on the All-WISE mid-infrared flux, which is known to correlate with the AGN bolometric luminosity (Richards et al. 2006), and the $2-10 \mathrm{keV}$ count rates of 5 archival mergers with similar WISE colors. We required a minimum of 10 counts in each observation to confirm the presence of X-ray point sources above the $3 \sigma$ level. The Chandra data were reduced using the Chandra Interactive Analysis of Observations (CIAO) software, version 4.7. Counts from each source were extracted from a circular region of radius $R=1$." 5 aperture. The background counts were extracted from regions

\footnotetext{
$\overline{15}$ http://www.mpa-garching.mpg.de/SDSS /
} 

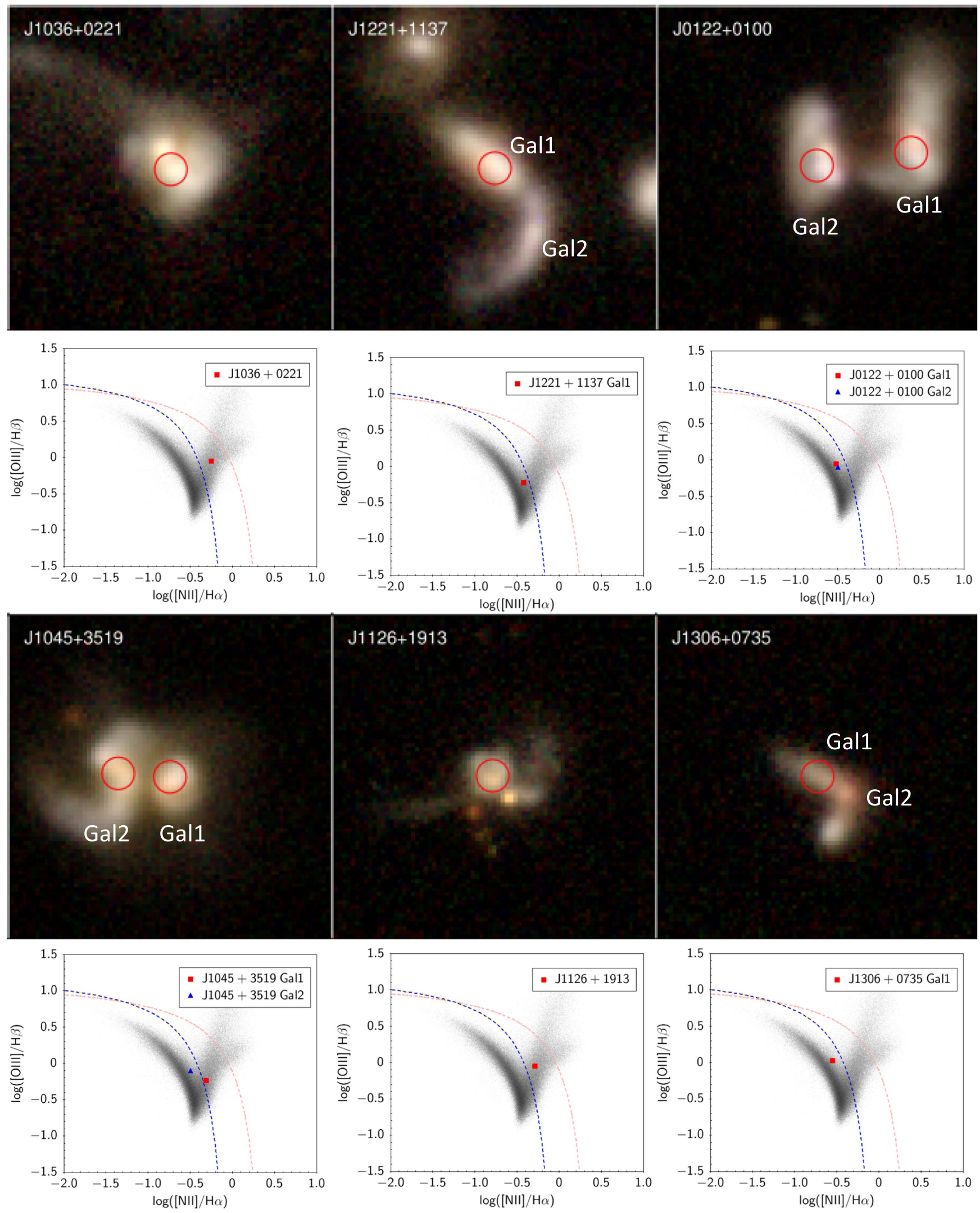

Figure 1. SDSS gri color composite images of the 6 Chandra targets. In each image, north is up and east is to the left. The SDSS fiber locations are indicated by the red circles, and the diameter of the circle corresponds to $3 \mathrm{arcsec}$. As can be seen, all systems are strongly disturbed, with separate nuclei resolvable by Chandra. We label the two galaxy nuclei listed in Table 4 in each image. Also shown under each image is the BPT diagram for the corresponding source showing the emission line ratios when optical spectra are available. The dotted blue and solid red line demarcations separating star-forming galaxies from AGNs are from Kauffmann et al. (2003) and Kewley et al. (2001), respectively. 
Table 1

WISE Merger Sample Properties

\begin{tabular}{|c|c|c|c|c|c|c|c|c|c|}
\hline $\begin{array}{l}\text { Name } \\
\text { (SDSS) }\end{array}$ & Redshift & $\begin{array}{c}D_{L} \\
(\mathrm{Mpc})\end{array}$ & $\underset{\left({ }^{\prime \prime}\right)}{\Delta \theta}$ & $\begin{array}{c}r_{p} \\
(\mathrm{kpc})\end{array}$ & $\log \left(M / M_{\odot}\right)_{1}$ & $\log \left(M / M_{\odot}\right)_{2}$ & $\underset{(\mathrm{mag})}{W 1-W 2}$ & $\underset{(\mathrm{mag})}{W 2-W 3}$ & $\begin{array}{c}L_{\mathrm{IR}} \\
10^{11} L_{\odot}\end{array}$ \\
\hline J012218.11+010025.7 & 0.05546 & 247.5 & 8.7 & 8.7 & 10.87 & 10.03 & 1.54 & 3.87 & 8.6 \\
\hline $\mathrm{J} 103631.88+022144.1$ & 0.05040 & 224.1 & 2.8 & 2.8 & 10.47 & $\ldots$ & 1.32 & 4.06 & 5.1 \\
\hline J112619.42+191329.3 & 0.10299 & 475.0 & 2.3 & 4.5 & 10.25 & $\ldots$ & 0.81 & 4.20 & $\cdots$ \\
\hline $\mathrm{J} 122104.98+113752.3$ & 0.06820 & 307.1 & 7.1 & 9.3 & 9.97 & $\ldots$ & 0.55 & 4.60 & 8.0 \\
\hline $\mathrm{J} 130653.60+073518.1$ & 0.11111 & 515.2 & 3.7 & 7.5 & 10.64 & $\ldots$ & 0.70 & 4.40 & 0.9 \\
\hline
\end{tabular}

Note. Column 3: Luminosity distance assuming a standard $\Lambda$ CDM cosmology with $H_{0}=70 \mathrm{~km} \mathrm{~s}^{-1} \mathrm{Mpc}^{-1}, \Omega_{\mathrm{M}}=0.3$, and $\Omega_{\Lambda}=0.7$. Columns 4-5: Approximate angular and physical projected spatial separation based on SDSS $r$-band images. Columns 6-7: Stellar mass of primary and secondary (when available) nuclei in each merger taken from the MPA/JHU catalog. Note for J122104.98+113752.3, we list the masses for the two sources in the MPA/JHU catalog, although the second mass listed above corresponds to the northern nucleus seen in Figure 1, which is not the location of the possible secondary Chandra nucleus listed in Table 4. Columns 8-9: WISE colors from the All-WISE data release of the WISE catalog. Column 10: 8-1000 $\mu \mathrm{m}$ IR luminosities calculated using the prescription from Sanders \& Mirabel (1996).

Table 2

Target and Chandra Observation Information

\begin{tabular}{|c|c|c|c|c|c|}
\hline $\begin{array}{l}\text { Name } \\
\text { (SDSS) }\end{array}$ & $\alpha$ & $\delta$ & $\begin{array}{l}\text { Chandra } \\
\text { Obs. Date }\end{array}$ & ObsID & $\begin{array}{l}\text { Exposure } \\
\quad(\mathrm{ks})\end{array}$ \\
\hline J012218.11+010025.7 & $01^{\mathrm{h}} 22^{\mathrm{m}} 18^{\mathrm{s}} \cdot 11$ & $+01^{\circ} 00^{\prime} 25^{\prime \prime} \cdot 76$ & 2014 Jun 29 & 16074 & 4.6 \\
\hline $\mathrm{J} 103631.88+022144.1$ & $10^{\mathrm{h}} 36^{\mathrm{m}} 31^{\mathrm{s}} .88$ & $+02^{\circ} 21^{\prime} 44^{\prime \prime} 10$ & 2014 Jul 04 & 16072 & 2.8 \\
\hline J112619.42+191329.3 & $11^{\mathrm{h}} 26^{\mathrm{m}} 19^{\mathrm{s}} \cdot 42$ & $+19^{\circ} 13^{\prime} 29^{\prime \prime} 35$ & 2015 Feb 03 & 16076 & 13.7 \\
\hline $\mathrm{J} 122104.98+113752.3$ & $12^{\mathrm{h}} 21^{\mathrm{m}} 04^{\mathrm{s}} .98$ & $+11^{\circ} 37^{\prime} 52^{\prime \prime} 34$ & 2014 Jul 10 & 16073 & 3.7 \\
\hline $\mathrm{J} 130653.60+073518.1$ & $13^{\mathrm{h}} 06^{\mathrm{m}} 53^{\mathrm{s}} \cdot 60$ & $+07^{\circ} 35^{\prime} 18^{\prime \prime} 18$ & 2014 Nov 20 & 16077 & 14.6 \\
\hline
\end{tabular}

Note. Columns 2-3: coordinates of the Chandra observations. Column 4: UT date of the Chandra/ACIS observations. Column 6: ACIS exposure time.

around the target regions free of spurious X-ray sources. All targets have low count rates, so pileup effects were insignificant. In Table 2, we list the details of the Chandra observations.

To determine the significance of source detections at the locations of the SDSS sources, we assumed binomial statistics and calculated the no-source probability, $P_{B}$, using the equation:

$$
P_{B}(X \geqslant S)=\sum_{k=S}^{N} \frac{N !}{X !(N-X) !} p^{X}(1-p)^{N-X}
$$

where $S$ is the total number of counts in the source extraction region in the full $(0.3-8 \mathrm{keV})$ band, $B$ is the total number of counts in the background extraction region, $N=S+B$, and $p=\frac{1}{1+\frac{A_{\mathrm{Bkg}}}{A_{\mathrm{src}}}}$, where $A_{\mathrm{Bkg}}$ and $A_{\mathrm{src}}$ are the background and source extraction apertures, respectively. $P_{B}$ is proportional to the probability that a source is spurious due to a background fluctuation (see Appendix A2 of Weisskopf et al. 2007).

Weighted Galactic total hydrogen column densities were derived using the Swift Galactic $N_{\mathrm{H}}$ tool, ${ }^{16}$ which is based on the work of Willingale et al. (2013) that appends the molecular hydrogen column density $N_{\mathrm{H} 2}$ to the atomic hydrogen column density $N_{\mathrm{H} \text { I }}$ from the Leiden-Argentine-Bonn (LAB) $21 \mathrm{~cm}$ survey (Kalberla et al. 2005).

\subsection{XMM-Newton}

We obtained an XMM-Newton observation of J0122+0100 (Observation ID: 0721900501; PI Satyapal) on 23 December 2013 as part of an unrelated program on AGNs in bulgeless galaxies.

\footnotetext{
${ }^{16}$ http://www.swift.ac.uk/analysis/nhtot/index.php
}

Since these data were available, we include the analysis of the XMM-Newton observation of $\mathrm{J} 0122+0100$ in this work. We calibrated our XMM-Newton event data using SAS, version 14.0.0, and using the latest current calibration files (CCFs). We performed all analyses of the pn $(C C D N R==4)$ and MOS CCDs $(\mathrm{CCDNR}==1)$, and kept all events with PATTERN between 0 and 4, for reliable spectral analysis. We further restricted our analysis to events between 0.3 and $12 \mathrm{keV}$. We searched for flaring particle background periods by making $10-12 \mathrm{keV}$ light curves on the source-subtracted event files, but we found no significant flaring periods. The effective exposure times for our final event files were 17.1 and $18.7 \mathrm{ks}$ for the pn and mos detectors, respectively.

We extracted counts from our event files by creating $0.3-10 \mathrm{keV}$ binned (bin factor $=32$ ) images of our event files, and using a circular source region with a radius $R=30^{\prime \prime}$ and a circular background region with radius $R=60^{\prime \prime}$ in a nearby source-free region. We obtained $239 \pm 21,64 \pm 11$, and $50 \pm 10$ background-subtracted source counts for pn, MOS 1, and MOS 2, respectively.

Using evselect, we created $0.3-10 \mathrm{keV}$ spectra for all three detectors using the same source and background regions, and we created redistribution matrix/ancillary response files using rmfgen/arfgen. Using GRPPHA, we grouped our spectra by a factor of 15 for the $\chi^{2}$ statistic. We performed our spectral analysis using XSPEC, version 12.8.1, and fitting the pn and MOS spectra simultaneously. ${ }^{17}$

\footnotetext{
${ }^{17}$ We do not attempt to combine the Chandra data with our XMM-Newton data, as there are not enough counts in the Chandra data to improve the statistics of the spectrum.
} 
Table 3

LBT Observation Log

\begin{tabular}{|c|c|c|c|c|c|}
\hline $\begin{array}{l}\text { Name } \\
\text { (SDSS) }\end{array}$ & $\begin{array}{c}L B T \\
\text { Obs. Date }\end{array}$ & $\begin{array}{c}\text { Total Exposure } \\
\text { (s) }\end{array}$ & $\begin{array}{c}\text { PA } \\
(\operatorname{deg})\end{array}$ & $\begin{array}{l}1^{\prime \prime}= \\
(\mathrm{kpc})\end{array}$ & $\begin{array}{l}\text { Aperture } \\
(\mathrm{kpc})\end{array}$ \\
\hline $\mathrm{J} 0122+0100 \mathrm{Gal}^{\mathrm{a}}$ & 2014 Nov 28 & 1800 & 0.0 & 1.07 & $1.60 \times 0.80$ \\
\hline $\mathrm{J} 0122+0100 \mathrm{Gal} 2^{\mathrm{a}}$ & 2014 Nov 28 & 600 & 0.0 & 1.07 & $1.60 \times 0.80$ \\
\hline $\mathrm{J} 1045+3519 \mathrm{Gal}^{\mathrm{a}}$ & 2015 Apr 02 & 1200 & 75.7 & 1.29 & $1.94 \times 0.97$ \\
\hline $\mathrm{J} 1045+3519 \mathrm{Gal} 2^{\mathrm{a}}$ & 2015 Apr 02 & 1200 & 75.7 & 1.29 & $1.94 \times 0.97$ \\
\hline $\mathrm{J} 1126+1913^{\mathrm{c}}$ & 2015 May 29 & 1200 & 320.0 & 1.89 & $2.84 \times 1.42$ \\
\hline $\mathrm{J} 1306+0735 \mathrm{Gal} 1^{\mathrm{b}}$ & 2015 Nov 17 & 1200 & 55.0 & 2.02 & $3.03 \times 1.52$ \\
\hline $\mathrm{J} 1306+0735 \mathrm{Gal} 2^{\mathrm{b}}$ & 2015 Nov 17 & 1440 & 155.0 & 2.02 & $3.03 \times 1.52$ \\
\hline
\end{tabular}

Notes. Columns 2: UT date of the LBT observations. Column 3: Exposure time. Column 4: Position angle of the slit. Column 5: Angular size to physical size conversion based on redshift and $\Lambda \mathrm{CDM}$ of $H_{\mathrm{o}}=70 \mathrm{~km} \mathrm{~s}^{-1} \mathrm{Mpc}^{-1}, \Omega_{\mathrm{M}}=0.3$ and $\Omega_{\lambda}=0.7$. Column 6: Spatial extraction aperture in kpc. This aperture is given as $X \times Y$, where $X$ is the slit width (either $1{ }^{\prime \prime} 0$ or $1 . .5$ ) and $Y$ is the spatial size extracted. $Y$ was selected based on the scientific needs and sky conditions of each observation. For both LUCI-1 and LUCI-2, 1 pixel $=0$." 25 . SDSS J1036+0735 was observed with a $1{ }^{\prime \prime} 0$ wide slit. All other targets were observed with a $1{ }^{\prime \prime} 5$ wide slit. The LUCI-1 1".0 longslit and LUCI-2 1". 5 longslits are 230" in length; The LUCI-1 1". 5 longslit is comprised of 3 segments, each $75^{\prime \prime}$ in length. The target galaxy or galaxies were observed using the central segment in the LUCI-1 1". 5 longslits.

${ }^{a}$ A single slit was used to measure both galaxies in a pair.

b Two different slit positions were used to observe the galaxy pair.

${ }^{c}$ Data obtained with LUCI-2; all other data obtained with LUCI-1.

\subsection{LBT Observations}

In order to search for obscured AGNs and to quantify the obscured star formation in each merger, we obtained nearinfrared ground based spectroscopy with the LBT LUCI (LBT Near Infrared Spectroscopic Utility with Camera Instruments) (Seifert et al. 2003, 2010) between 2014 November 28 and 2015 November 17. The spectra of the six mergers were taken with the N1.8 camera, centered on the coordinates of the X-ray detections listed listed in Table 3. We used the G200 grating with the HKspec filter with a 1".5 wide longslit for all targets except for J1036+0221 where a slit width of 1!"0 was employed instead. The LUCI-1 imager/spectrograph was used for all objects but J1126+1913 where LUCI-2 was used instead. The LUCI-1 1".0 longslit and LUCI-2 1." 5 longslit are $230^{\prime \prime}$ in length. The LUCI-1 1".5 longslit is comprised of 3 segments, each being $75^{\prime \prime}$ in length. The target galaxy or galaxies were observed using the central segment in the LUCI1 1"' 5 longslits. For both LUCI-1 and LUCI-2, 1 pixel $=0$ "' 25 . Observations were done using an $\mathrm{AB}$ pattern of nodding along the slit. AOV-type stars at similar air masses were observed before the target to remove telluric features. Using calibration $\mathrm{Ne}$ and $\mathrm{Ar}$ arc lines, we measured an average spectral resolution of $0.0019-0.0025 \mu \mathrm{m}$ per pixel, or $R \sim 858-1376$ over this wavelength range.

Spectral extraction and wavelength and flux calibration were performed using a set of custom IRAF scripts following the general procedures described in (Satyapal et al. 2016). In particular, the one-dimensional spectra were extracted with apall using a 3 pixel diameter aperture for all spectra except for $\mathrm{J} 1221+1137$, where a 4 pixel aperture was used for an increased signal-to-noise ratio. The choice for the slit and extraction aperture size was based on the sky conditions of each observations and the signal-to-noise ratio predicted to allow measurements of emission and absorption features of interest for this study. Table 3 lists the observing details for the LBT observations, including the spatial extraction aperture size in kiloparsecs, given as $X \times Y$, where $X$ is the slit width (either 1 ." 0 or 1 !" 5 converted to a spatial size) and $Y$ is the extraction aperture in metric sizes. For J0122+0100, J1221+1137, and J1306+0735 two slit positions were used to observe the two individual galaxies. The two galaxies of the J1045+3519 pair were caught in one single slit at position angle $\mathrm{PA}=75^{\circ} .7$. For the objects $\mathrm{J} 1036+0221$ and $\mathrm{J} 1126+1913$, a single slit position centered on the single Chandra detection was used.

Flux calibration and removal of Telluric features was performed using the generalized version of the SPEX XTELLCOR software (Vacca et al. 2003). The final telluric corrected and flux calibrated data have been modeled and measured with specfit (Kriss 1994), a method that employs line and continuum spectral fitting via an interactive $\chi^{2}$ minimization. The formal errors on the line fluxes include errors in the continuum subtraction and flux calibration, and have been then propagated to the line ratios. For each of the fitted spectral range the continuum was approximated by a linear fit, while the emission features were modeled by Gaussian profiles. Figures 4-6 show these 1D near-infrared spectra, along with a zoomed-in region of the $\mathrm{Pa} \alpha$, corresponding to the position of the Chandra sources for each target. The spectral measurements and their physical interpretation are discussed in Section 4.2.

\section{Results \\ 4.1. X-Ray Results \\ 4.1.1. Chandra ACIS Imaging Results}

In Figure 2, we show the Chandra $0.3-8 \mathrm{keV}$ images with SDSS contours overlaid for the six mergers in our sample. In Table 4 we list the number of counts detected in the $0.3-8 \mathrm{keV}$ and $2-8 \mathrm{keV}$ bands, and list the detection threshold, $P_{B}$, associated with each position. There are a number of $P_{B}$ thresholds employed in the literature to define source detection significance. Based on weak sources in Chandra deep field images, the threshold for $P_{B}$ adopted based on a balance between reliability and completeness varies from 0.002 to 0.007 (Xue et al. 2011, 2016; Luo et al. 2017). Based on even the lowest of all these thresholds, all sources are detected. Note 

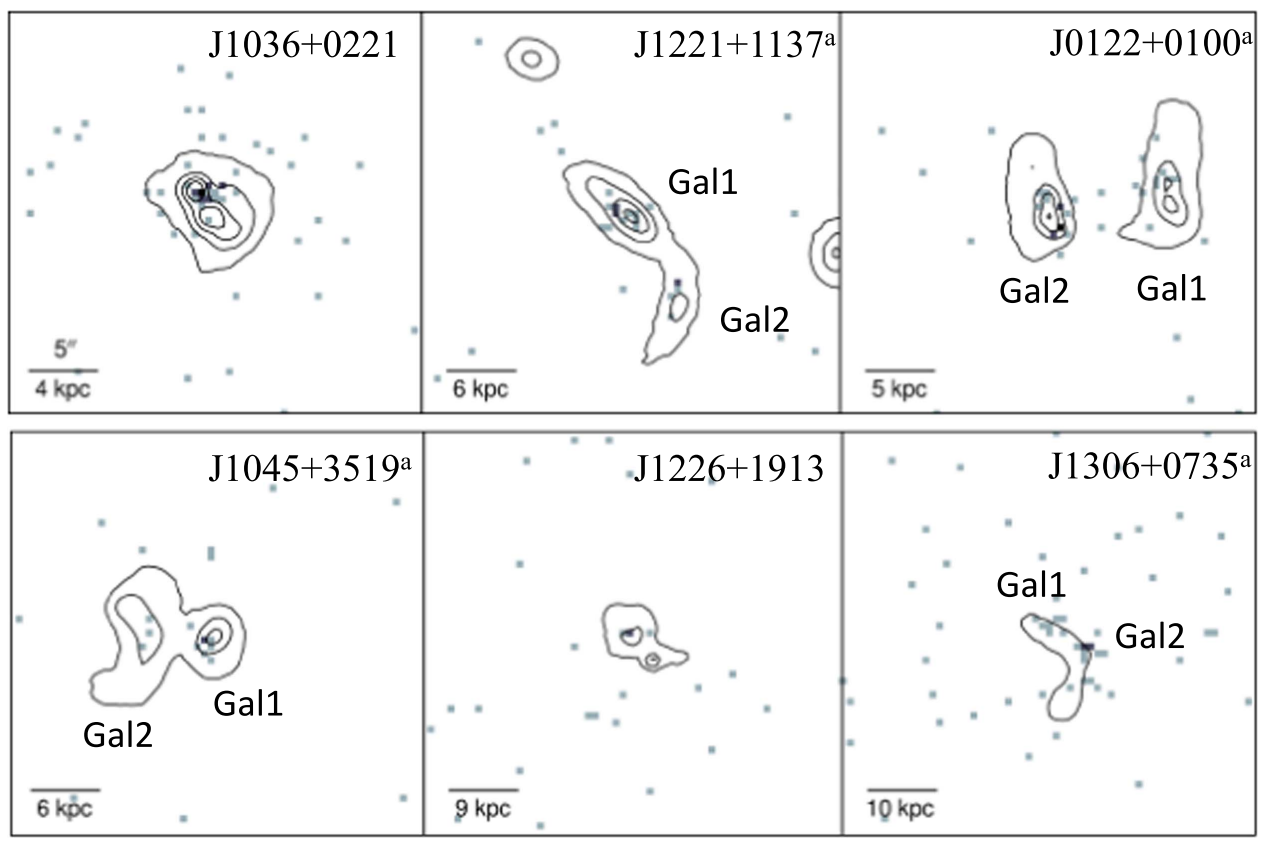

Figure 2. $0.3-8 \mathrm{keV}$ images with SDSS $r$-band contours overlaid for each target. a-dual X-ray sources. North is up and east is to the left.

that the detection thresholds adopted in the literature for sources at known locations are often $P_{B}<0.01$, which corresponds to $2.6 \sigma$ (e.g., Lansbury et al. 2014). We note that due to insufficient counts, coupled with the fact the exact location of the galaxy nuclei is uncertain in the advanced mergers in our sample (see SDSS images in Figure 1), it is impossible to quantify any possible spatial offsets between the positions of the detected Chandra sources and the actual galactic nuclei. It is therefore not possible to determine using these observations alone if some of the mergers host offset AGNs, another unambiguous signature of a galaxy merger that can probe AGN triggering through galaxy interactions (Barrows et al. 2016, 2017). We note that for sources within the $2^{\prime}$ of the boresight, the absolute accuracy of source locations on the ACIS-S chip has a $90 \%$ uncertainty radius of $\sim 0$. $6,{ }^{18}$ indicating that the positions of all detected targets are consistent with the locations of the SDSS knots, suggesting that the detected sources likely correspond to the nuclei of the mergers. J1036+0221, J1045+3519 Gal 1, J1221+1137 Gal 1, and $\mathrm{J} 1306+0735 \mathrm{Gal} 2$ were detected in the hard band using the same detection threshold $P_{B}<0.002$ using the $2-8 \mathrm{keV}$ source counts. Note that there are insufficient counts to perform a spectral analysis or to obtain reliable HRs for our targets. We therefore list in Table 6 the observed X-ray luminosity, assuming a simple power-law model with $\Gamma=1.8$, corrected for Galactic absorption.

\subsubsection{XMM-Newton Results}

We fit the X-ray spectrum of J0122+0100 between 0.3 and $10 \mathrm{keV}$ with a simple power-law model with Galactic absorption. To account for inter-detector sensitivity differences, we appended a constant value to each detector group, fitting the MOS detectors relative to pn (setting the pn constant equal to unity), but otherwise tying the groups' model parameters. Explicitly, the model we employ is const*phabs*zpow, where for the Galactic absorption term phabs we adopt

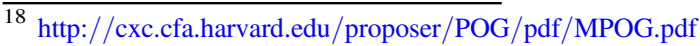

$3.5 \times 10^{20} \mathrm{~cm}^{-2}$ as per Section 3.1. We find a good fit to the data $\left(\chi^{2} /\right.$ dof $\left.=21.07 / 22\right)$ with a power-law index of $\Gamma=2.05 \pm 0.2$, typical of AGNs. We note that we do not find any requirement for an additional intrinsic absorber, and the observed 2-10 keV luminosity after correcting for Galactic absorption is $\log \left(L_{2-10 \mathrm{keV}} / \mathrm{erg} \mathrm{s}^{-1}\right)=41.1 \pm 0.1$.

However, our spectral fit is also consistent with a Comptonthick AGN with some fraction of X-ray photons scattered back into the line of sight at radii larger than the absorbing medium by an optically thin, ionized medium. Using a Gaussian model component with $\sigma=0.1 \mathrm{keV}$, we find a $90 \%$ upper limit on the equivalent width (EW) of the $\mathrm{Fe} \mathrm{K} \alpha$ line of $\sim 3 \mathrm{keV}$. This is unconstrained enough to allow the possibility that the line-ofsight absorber could have a column density as high as $N_{\mathrm{H}}=10^{25} \mathrm{~cm}^{-2}$ (e.g., Murphy \& Yaqoob 2009). This being the case, the intrinsic X-ray luminosity could be a factor of 100 or higher (e.g., Ueda et al. 2007). We show the X-ray spectrum of $\mathrm{J} 0122+0100$ in Figure 3 .

\subsection{Near-infrared Spectra}

In Figures 4-6, we plot the 1D near-infrared spectra corresponding to the position of the Chandra sources for each target. We detect a plethora of emission lines, including a prominent $\mathrm{Pa} \alpha$ line, the $\mathrm{Br} \gamma$ line, the [Fe II] $1.644 \mu \mathrm{m}$ line, several molecular hydrogen lines, and the [Si VI] coronal line at $1.963 \mu \mathrm{m}$ in several targets. $\mathrm{J} 1036+0221$ is the only system that shows a [Si X] $\lambda 1.43 \mu \mathrm{m}$ detected at the $2.6 \sigma$ level. In several of these systems we detected and measured the $\mathrm{CO}$ $1.6 \mu \mathrm{m}$ absorption band, whose EW we use here for constraining the age of the stellar populations associated with each nucleus.

We searched for AGN signatures in the galaxy nuclei by exploring the possibility of detecting either a broad recombination line emission component or a coronal line. None of the spectral fits were consistent with the presence of such a broad component to the recombination line emission. For the spectra of J0122+0100 Gal 1 and Gal 2, J1221+1137 Gal 1, J1306 $+0735 \mathrm{Gal} 1, \mathrm{~J} 1036+0221$, and $\mathrm{J} 1126+1913$ we have 
Table 4

Chandra X-Ray Detections

\begin{tabular}{|c|c|c|c|c|c|c|c|c|}
\hline \multirow{2}{*}{$\begin{array}{l}\text { Name } \\
\text { (SDSS) }\end{array}$} & \multirow[b]{2}{*}{ Source } & Galaxy & Galactic & \multirow[b]{2}{*}{$\alpha_{\mathrm{X}}$} & \multirow[b]{2}{*}{$\delta_{\mathrm{X}}$} & \multirow{2}{*}{$\begin{array}{c}\text { Count } \\
0.3-8 \mathrm{keV}\end{array}$} & \multirow{2}{*}{$\begin{array}{l}\text { Count } \\
2-8 \mathrm{keV}\end{array}$} & \multirow[t]{2}{*}{$\log P_{\mathrm{B}}$} \\
\hline & & Nucleus & $N_{\mathrm{H}}$ & & & & & \\
\hline J0122+0100 & $\mathrm{NW}^{\mathrm{a}}$ & Gal1 & & $01^{\mathrm{h}} 22^{\mathrm{m}} 17^{\mathrm{s}} .606$ & $+01^{\circ} 00^{\prime} 28^{\prime \prime} 44$ & $6 \pm 2$ & $1 \pm 1$ & -9.7 \\
\hline $\mathrm{J} 1036+0221$ & & & 3.37 & $10^{\mathrm{h}} 36^{\mathrm{m}} 31^{\mathrm{s}} \cdot 920$ & $+02^{\circ} 21^{\prime} 45^{\prime \prime} 66$ & $17 \pm 4$ & $8 \pm 3$ & -31.5 \\
\hline \multirow{2}{*}{$\mathrm{J} 1045+3519$} & W & Gal1 & 1.96 & $10^{\mathrm{h}} 45^{\mathrm{m}} 18^{\mathrm{s}} .087$ & $+35^{\circ} 19^{\prime} 12^{\prime \prime} 41$ & $8 \pm 3$ & $3 \pm 2$ & -10.9 \\
\hline & $E^{a}$ & Gal2 & & $10^{\mathrm{h}} 45^{\mathrm{m}} 18^{\mathrm{s}} \cdot 437$ & $+35^{\circ} 19^{\prime} 13^{\prime \prime} 51$ & $3 \pm 2$ & $0 \pm 0$ & -4.0 \\
\hline $\mathrm{J} 1221+1137$ & $\mathrm{SW}^{\mathrm{a}}$ & Gal2 & & $12^{\mathrm{h}} 21^{\mathrm{m}} 04^{\mathrm{s}} .776$ & $+11^{\circ} 37^{\prime} 47^{\prime \prime} 43$ & $4 \pm 2$ & $0 \pm 0$ & -5.8 \\
\hline \multirow[t]{2}{*}{$\mathrm{J} 1306+0735$} & SW & Gal2 & 2.51 & $13^{\mathrm{h}} 06^{\mathrm{m}} 53^{\mathrm{s}} \cdot 429$ & $+07^{\circ} 35^{\prime} 17^{\prime \prime} 17$ & $10 \pm 3$ & $6 \pm 2$ & -12.2 \\
\hline & $\mathrm{NE}^{\mathrm{a}}$ & Gal1 & & $13^{\mathrm{h}} 06^{\mathrm{m}} 53^{\mathrm{s}} \cdot 601$ & $+07^{\circ} 35^{\prime} 18^{\prime \prime} 85$ & $7 \pm 3$ & $0 \pm 0$ & -7.7 \\
\hline
\end{tabular}

Note.

${ }^{\text {a }}$ Source/extraction aperture position calculated by centroiding the source on the smoothed $0.3-8 \mathrm{keV}$ image using a 3-pixel Gaussian kernel. All other source positions calculated using wavdetect; $N_{\mathrm{H}}$ is Galactic and in units of $\times 10^{20} \mathrm{~cm}^{-2}$. Note that the energy ranges for the counts correspond to rest-frame energies. Column 9 lists the logarithm of the no-source probability, $P_{B}$, which is the probability of still observing the same number of source counts or more under the assumption that there is no real source at the SDSS location and that the observed excess number of counts over background is purely due to background fluctuations. Values below $P_{B}<0.002\left(\log P_{\mathrm{B}}<-2.7\right)$ are considered in this work as a detection.

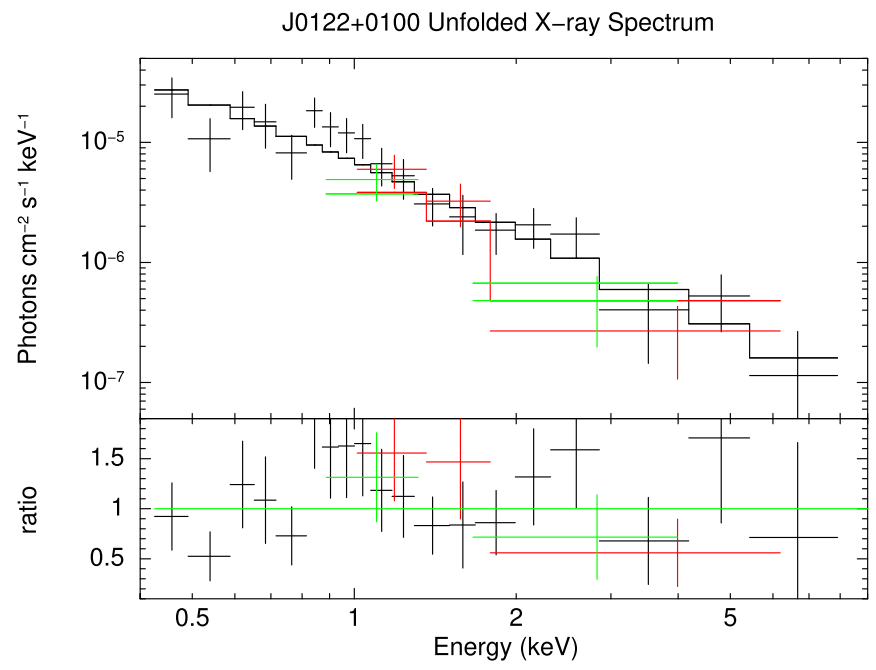

Figure 3. The best-fit power-law model from Section 3.2 of J0122+0100 from the XMM-Newton data. The black, red, and green lines correspond to the pn, MOS1, and MOS2 spectra, respectively.

identified redshifted and/or blueshifted wings in the $\mathrm{Pa} \alpha$ emission (indicated as $\mathrm{Pa} \alpha-\mathrm{b}$ and $\mathrm{Pa} \alpha-\mathrm{r}$ in the spectra of Figures 4-6), for which simultaneous multiple Gaussian fits provided a significantly better $\chi^{2}$ than in the case of using only one single component for the main $\operatorname{Pa} \alpha$ feature. We are investigating the physical origin of these features, whether related to gas rotation, outflows, or winds, in a separate paper that discusses in detail the near-infrared spectral properties of a larger sample of WISE-selected advanced mergers (A. Constantin et al. 2017, in preparation). The $\operatorname{Pa} \alpha$ flux and EW values used in this analysis refer to the main feature alone. The additional flux contributions from the wings amount to less than $14 \%$, thus they do not affect the overall conclusions of this work. We do detect coronal line emission in several targets, which we discuss in Section 5.

In Table 5, we list the recombination line fluxes, and the derived extinction, assuming case B recombination with an intrinsic $\mathrm{Pa} \alpha$ to $\mathrm{Br} \gamma$ flux ratio of 12.5 (Osterbrock \& Ferland 2006) assuming the extinction curve from Landini et al. (1984).
Our near-infrared spectra enables an extinction-insensitive determination of the SFR, if we make the assumption that all of the near-infrared recombination line flux is originating solely from gas ionized by young stars. In reality, some of the recombination line flux can potentially originate from the narrow line gas ionized by any putative AGN. The derived SFRs are therefore upper limits to the total SFR expected from each source. The SFR was estimated using the relation between the SFR and the H $\alpha$ flux from Kennicutt et al. (1994), assuming an intrinsic $\mathrm{H} \alpha$ to $\mathrm{Pa} \alpha$ line flux ratio of 7.82 for galaxies with $12+\log (\mathrm{O} / \mathrm{H})>8.35$ (Osterbrock \& Ferland 2006).

\section{The Nature of the Nuclear Sources}

Our Chandra observations reveal X-ray sources detected in all of the six mergers in our sample, with four of them showing dual $\mathrm{X}$-ray sources coincident with the galaxy optical nuclei (see Figure 2), suggesting that mid-infrared selection is an effective pre-selection strategy for identifying nuclear X-ray point sources in advanced mergers. In Table 6 , we list the derived observed X-ray luminosity (uncorrected for intrinsic absorption) of each detected source. In all cases, there are insufficient counts to fit the X-ray spectrum and constrain the intrinsic absorption and characterize the hardness of the nuclear source. The observed hard X-ray luminosities of the nuclear sources range from $L_{2-10 \mathrm{keV}} \sim 4 \times 10^{40} \mathrm{erg} \mathrm{s}^{-1}$ to $\sim 2 \times 10^{41} \mathrm{erg} \mathrm{s}^{-1}$. These observed X-ray luminosities are within the range of the observed hard X-ray luminosities reported in the literature for confirmed dual AGNs (see Table 8; for references to X-ray luminosities, see (Komossa et al. 2003; Brassington et al. 2007; Bianchi et al. 2008; Comerford et al. 2011; Koss et al. 2011, 2012; Teng et al. 2012; Liu et al. 2013; Comerford et al. 2015)). While X-ray emission coincident with the nuclei in interacting galaxies is highly suggestive of the presence of an AGN, X-ray emission can also arise purely from stellar processes. In this section, we combine mid-infrared, X-ray, and near-infrared spectral diagnostics to explore the nature of the nuclear sources in the advanced mergers and determine if they require the presence of an AGN. Based on this combined analysis, we present a 

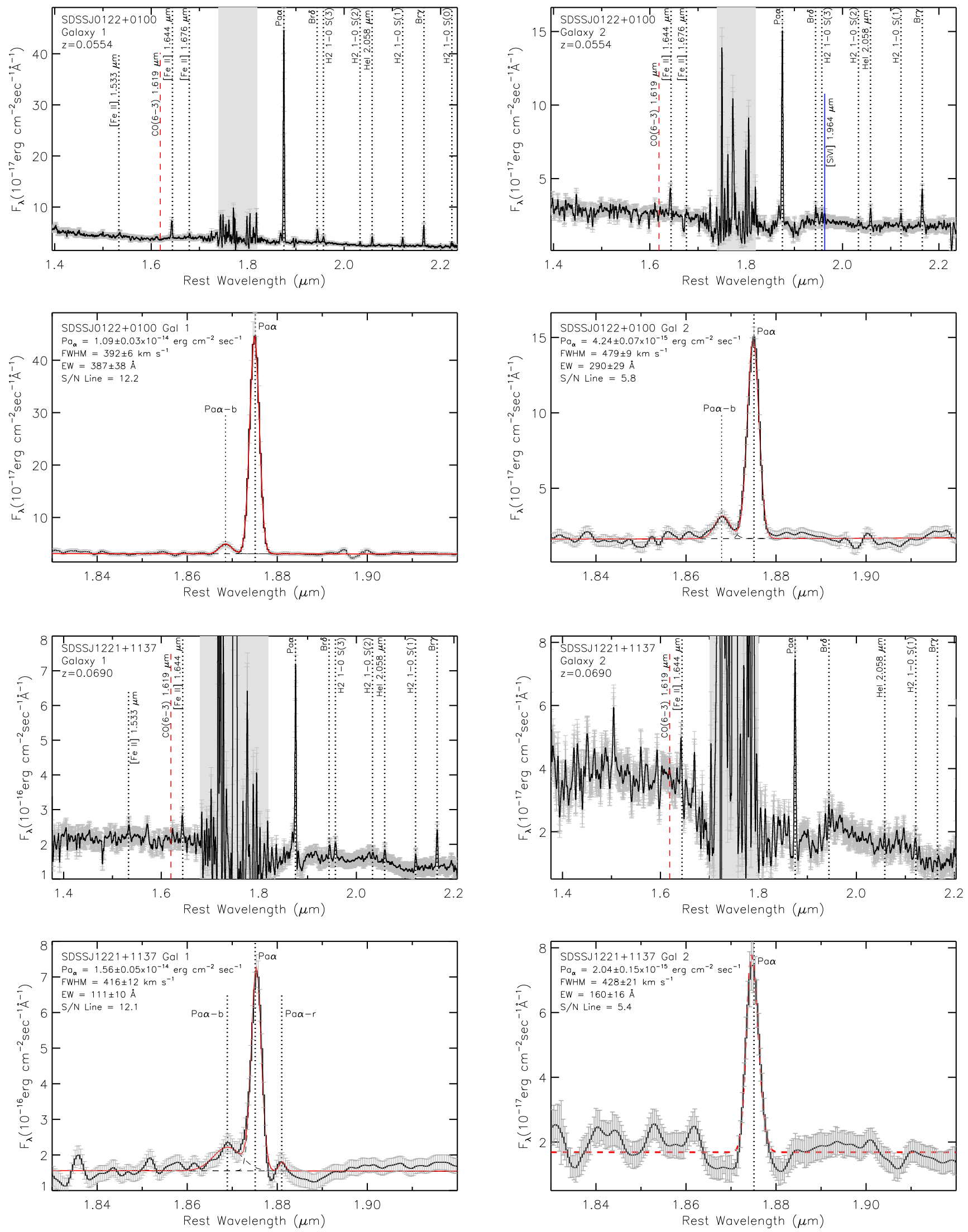

Figure 4. The LBT LUCI near-infrared spectra of J0122+0100 and J1221+1137 centered on each Chandra source corrected for redshift, with labels for the features detected. The vertical gray shaded area represents a region of strong atmospheric telluric absorption between the $\mathrm{H}$ and $\mathrm{K}$ bands $(\simeq 1.8 \mu \mathrm{m}-1.95 \mu \mathrm{m})$. Under each full spectrum is the segment of the spectrum centered around the $\mathrm{Pa} \alpha$ line. 

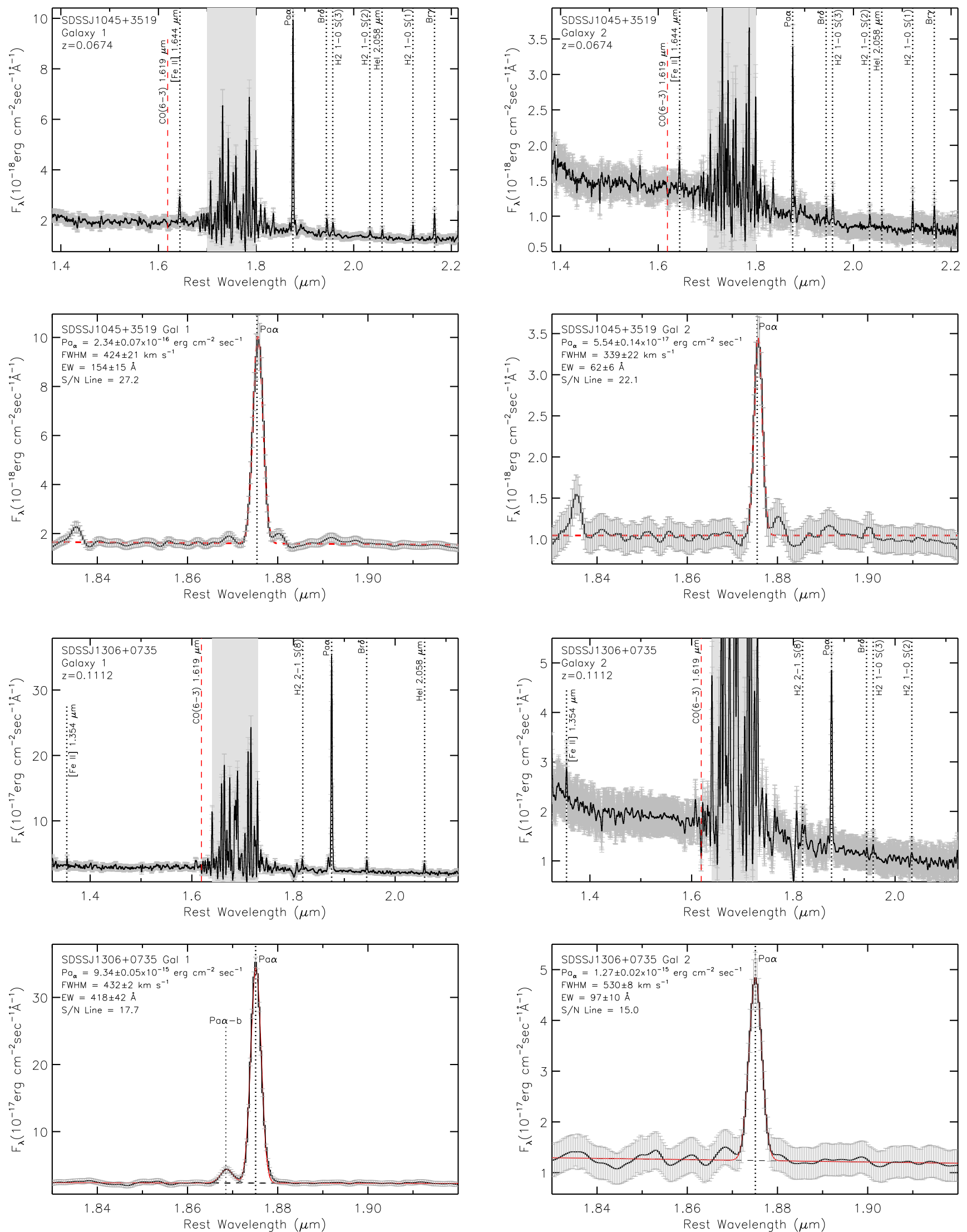

Figure 5. The LBT LUCI near-infrared spectra of J1045+3519 and J1306+0735 centered on each Chandra source corrected for redshift, with labels for the features detected. The vertical gray shaded area represents a region of strong atmospheric telluric absorption between the $\mathrm{H}$ and $\mathrm{K}$ bands $(\simeq 1.8 \mu \mathrm{m}-1.95 \mu \mathrm{m})$. Under each full spectrum is the segment of the spectrum centered around the $\mathrm{Pa} \alpha$ line. 

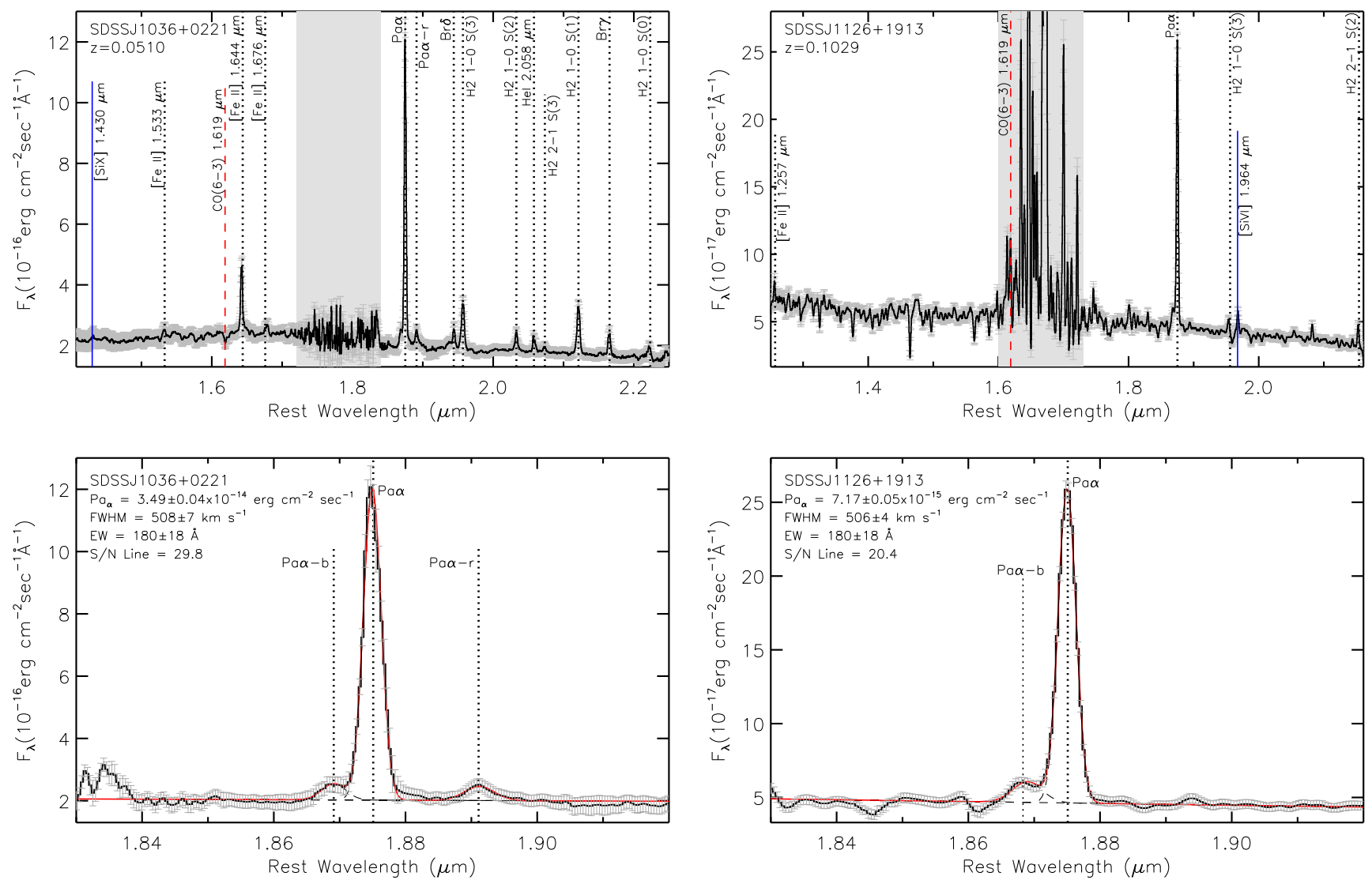

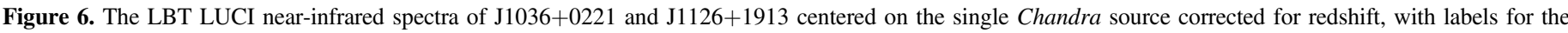

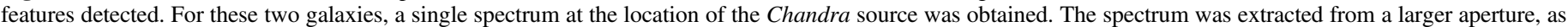

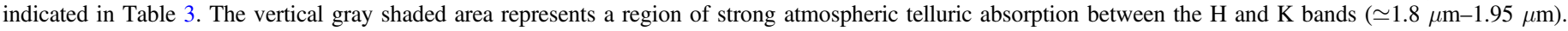
Under each full spectrum is the segment of the spectrum centered around the $\mathrm{Pa} \alpha$ line.

summary of all AGN diagnostics and our adopted classification for each of the advanced mergers in the sample.

\subsection{The Contribution from X-Ray Binaries}

We consider the possibility that the nuclear X-ray emission results from the integrated emission from a population of X-ray binaries within the Chandra extraction aperture. Using the SFR calculated using the near-infrared recombination line fluxes, we can estimate the X-ray emission expected from stellar processes alone in order to determine if an additional source of X-ray emission from an AGN is required. Given the SFRs of the galaxies in our sample, high-mass X-ray binaries (HMXBs) are expected to dominate the XRB population (Gilfanov 2004). If HMXBs do indeed make a significant contribution to the X-ray emission, the underlying nuclear stellar population in the host galaxies must be dominated by a young stellar population, when the population of HMXBs is expected to be high. Our near-infrared spectra allow us to test this hypothesis by constraining the ages of the nuclear stellar populations. The $H$-band is dominated by the presence of stellar absorption lines indicative of late-type giants and red supergiants, the strongest of which is the $\mathrm{CO}$ (6-3) transition at $1.6189 \mu \mathrm{m}$. The depth of the CO bandhead is known to vary with age and metallicity, providing a way to constrain the ages of the stellar populations (e.g., Oliva et al. 1995; Origlia et al. 1997; Origilia et al. 1998).

Following the procedure described in Satyapal et al. (2016), we compared the observed EWs of the CO bandhead with the Maraston \& Strömbäck (2011; hereafter M11) set of intermediate-high resolution stellar population models. In Figure 7, we plot the CO EWs for three different M11 instantaneous starburst models corresponding to Kroupa, Chabrier, and Salpeter initial mass functions (IMFs), as a function of time (see Satyapal et al. (2016) for details). The horizontal dashed lines indicate the observed EWs for the targets in our sample. As can be seen, the observed CO (6-3) bandhead for our targets imply relatively young stellar populations $(t<20 \mathrm{Myr})$. In addition to the $\mathrm{CO}$ bandhead, the EWs of hydrogen recombination lines are strongly dependent on age, showing a steep decline as the most massive stars evolve off the main sequence, causing a simultaneous decrease in the ionizing photon flux and an increase in the $K$-band continuum flux. We also plot in Figure 7 the $\operatorname{Br} \gamma \mathrm{EW}$ as a function of age using the Starburst 99 star formation models (Leitherer et al. 1995, 2014). As can be seen, the observed EWs, when available, are also consistent with a young stellar population, with ages $\approx 7-8 \mathrm{Myr}$. While these ages are relatively young, they are beyond the age at which the HMXB population peaks. For solar metallicity galaxies, the peak in the number of bright HMXBs $\left(L_{X}>10^{39} \mathrm{erg} \mathrm{s}^{-1}\right.$ is approximately 5 Myr after the burst (see Figure 1 in Linden et al. 2010), and drops precipitously to below $1 \mathrm{HMXBs}$ at $7 \mathrm{Myr}$ for a starburst of $10^{6} M_{\odot}$, approximately a factor of 3.5 times lower than the number of less luminous HMXBs. Thus, while the nuclear stellar populations are likely to be relatively young, it is unlikely that all of the observed X-ray emission is due to a population of HMXBs in our sample. 
Table 5

Near-infrared Hydrogen Recombination Line Measurements

\begin{tabular}{|c|c|c|c|}
\hline $\begin{array}{l}\text { Name } \\
\text { (SDSS) }\end{array}$ & $\begin{array}{c}\mathrm{Pa} \alpha \text { Flux } \\
\left(10^{-15} \mathrm{erg} \mathrm{cm}^{-2} \mathrm{~s}^{-1}\right)\end{array}$ & $\begin{array}{c}\text { Br } \gamma \text { Flux } \\
\left(10^{-16} \mathrm{erg} \mathrm{cm}^{-2} \mathrm{~s}^{-1}\right)\end{array}$ & $\begin{array}{c}A_{V} \\
(\mathrm{mag})\end{array}$ \\
\hline J0122+0100 Gal 1 & $10.9 \pm 0.3$ & $11.3 \pm 0.3$ & $3.49 \pm 0.06$ \\
\hline $\mathrm{J} 0122+0100 \mathrm{Gal} 2$ & $4.24 \pm 0.07$ & $9.4 \pm 0.4$ & $12.98 \pm 0.05$ \\
\hline $\mathrm{J} 1036+0221$ & $34.9 \pm 0.4$ & $33.2 \pm 0.9$ & $11.82 \pm 0.04$ \\
\hline $\mathrm{J} 1045+3519$ Gal 1 & $0.23 \pm 0.07$ & $0.3 \pm 0.03$ & $6.81 \pm 0.43$ \\
\hline $\mathrm{J} 1045+3519$ Gal 2 & $0.06 \pm 0.01$ & $0.1 \pm 0.02$ & $7.33 \pm 0.35$ \\
\hline $\mathrm{J} 1126+1913$ & $7.17 \pm 0.05$ & $\cdots$ & $\cdots$ \\
\hline $\mathrm{J} 1221+1137 \mathrm{Gal} 1$ & $15.6 \pm 0.5$ & $36.4 \pm 1.1$ & $\cdots$ \\
\hline $\mathrm{J} 1221+1137 \mathrm{Gal} 2$ & $2.0 \pm 0.1$ & $0.9 \pm 0.2$ & $15.03 \pm 0.06$ \\
\hline $\mathrm{J} 1306+0735 \mathrm{Gal} 1$ & $9.34 \pm 0.05$ & $\cdots$ & $\cdots$ \\
\hline $\mathrm{J} 1306+0735$ Gal 2 & $1.27 \pm 0.02$ & $\ldots$ & $\ldots$ \\
\hline
\end{tabular}

Note. Column 4 lists the extinction derived from the near-infrared recombination line ratio assuming Case B recombination (see Section 4.2 for details).

Table 6

Nuclear Star Formation Rates and Predicted X-Ray Luminosities from XRBs

\begin{tabular}{lccr}
\hline \hline $\begin{array}{l}\text { Name } \\
(\text { SDSS })\end{array}$ & $\begin{array}{c}\text { SFR } \\
\left(M_{\odot} \mathrm{yr}^{-1}\right)\end{array}$ & $\begin{array}{c}L_{2-10 \mathrm{keV}}^{\mathrm{SF}} \\
\left(10^{40} \mathrm{erg} \mathrm{s}^{-1}\right)\end{array}$ & \multicolumn{1}{c}{$\begin{array}{c}L_{2-10 \mathrm{keV}}^{\text {Obs. }} \\
\left(10^{40} \mathrm{erg} \mathrm{s}^{-1}\right)\end{array}$} \\
\hline J0122+0100 Gal 1 & 4.86 & $0.79_{-0.36}^{+1.73}$ & $5.87 \pm 1.96$ \\
J0122+0100 Gal 2 & 1.85 & $0.38_{-0.87}^{+0.83}$ & $5.87 \pm 2.93$ \\
J1036+0221 & 0.16 & $0.03_{-0.01}^{+0.07}$ & $22.42 \pm 5.27$ \\
J1045+3519 Gal 1 & 0.16 & $0.42_{-0.19}^{+0.92}$ & $11.83 \pm 4.44$ \\
J1045+3519 Gal 2 & 0.04 & $0.006_{-0.003}^{+0.013}$ & $4.44 \pm 2.96$ \\
J1126+1913 & 12.86 & $2.08_{-0.95}^{+4.45}$ & $4.84 \pm 2.42$ \\
J1221+1137 Gal 1 & 11.28 & $1.93_{-0.88}^{+4.22}$ & $20.86 \pm 5.69$ \\
J1221+1137 Gal 2 & 1.42 & $0.90_{-0.41}^{+1.97}$ & $7.50 \pm 3.75$ \\
J1306+0735 Gal 1 & 18.85 & $3.21_{-1.47}^{+7.02}$ & $9.36 \pm 2.80$ \\
J1306+0735 Gal 2 & 1.45 & $0.02_{-0.01}^{+0.04}$ & $13.37 \pm 5.73$ \\
\end{tabular}

Note. Column 2 lists the SFR calculated using the extinction corrected $\mathrm{Pa} \alpha$ line flux (see Section 4.2 for details). Column 3 lists the predicted 2-10 keV luminosity from stellar processes using the (Lehmer et al. 2010) relation (see Section 5.1 for details). The uncertainties correspond to the 0.34 dex scatter in the relation presented in (Lehmer et al. 2010). Column 4 lists the observed 2-10 keV luminosity uncorrected for intrinsic absorption. Note that this is a lower limit, since the X-ray luminosities are not corrected for any intrinsic absorption.

We calculated the expected X-ray luminosity from XRBs using our near-infrared data. Using the extinction-corrected $\operatorname{Pa} \alpha$ flux, we estimated (see Section 4.2) and list in Table 6 the SFR at the location of each nucleus, assuming that all of the $\mathrm{Pa} \alpha$ flux arises in gas ionized only by the stellar component. To calculate the predicted X-ray emission from XRBs, we used the global galaxy-wide relationship between stellar mass, SFR, and X-ray emission given in Lehmer et al. (2010), which was derived using a sample of local LIRGs with similar infrared luminosities as our targets. As can be seen from Table 6, the observed 2-10 keV luminosities for all targets in the sample are above values predicted from XRBs from the Lehmer et al. (2010) relation, taking into account the 0.34 dex scatter in the relation. We performed a $\mathrm{K}-\mathrm{S}$ test on the distributions of observed X-ray luminosities and the predicted luminosities from XRBs listed in Table 3 and found that the probability that the luminosities come from the same distribution is only $1.9 \times 10^{-5} \%$, strongly suggesting that the X-ray emission is not due to star formation for the sample as a whole. Moreover, the WISE color selection of our sample suggests the presence of at least one AGN in all our targets.

\subsection{ULX Origin for the X-Ray Emission}

We also consider the possibility that the $\mathrm{X}$-ray sources are ultraluminous X-ray sources. ULXs are off-nuclear X-ray sources with luminosities in excess of $10^{39} \mathrm{erg} \mathrm{s}^{-1}$, which is the Eddington luminosity of a $10 M_{\odot}$ stellar mass black hole. The luminosities of ULXs can be produced either by anisotropic emission (beaming) or super Eddington accretion from a stellar sized black hole or by accretion onto intermediate mass black holes (IMBHs), although evidence for the latter scenario is sparse (for the most recent review see Feng \& Soria 2011). Although ULXs are generally rare, they are preferentially found in regions of enhanced star formation (e.g., Gao et al. 2003; Mapelli et al. 2008). Since our targets are advanced mergers with significant star formation, the possibility that the detected X-ray sources are ULXs associated with stellar-sized black holes must be considered.

While a ULX origin for the X-ray detections is a possibility, the vast majority of ULXs have total intrinsic $0.2-10 \mathrm{keV}$ luminosities between $10^{39}-10^{40} \mathrm{erg} \mathrm{s}^{-1}$ (Sutton et al. 2012), significantly below the observed luminosities of our targets, which are themselves lower limits to the actual absorptioncorrected luminosities. Using the comprehensive catalog of ULXs by Walton et al. (2011), there are only 7 out of 655 ULXs with luminosities above $10^{40} \mathrm{erg} \mathrm{s}^{-1}$. Recent follow-up observations of these so-called hyper-luminous ULXs (HLXs) have demonstrated a growing number that are likely background AGNs (Zolotukhin et al. 2016) or stripped nuclei of dwarf galaxies during mergers (Soria et al. 2013), calling into question the existence of any bona fide off-nuclear X-ray sources with luminosities comparable to the lower limit implied by our detections. Moreover, the WISE colors at the location of the detected X-ray sources in our sample are extremely red, strongly favoring an AGN origin for the X-ray detections. Indeed, ULXs are not associated with red, AGN-like midinfrared colors, as shown in Secrest et al. (2015b). The average $W 1-W 2$ color associated with ULXs is 0.07 , and corresponds to the colors of the underlying host galaxy. Of the 655 input galaxies from the Walton catalog, 231 were bright enough to have extended WISE photometry; of those, only 7 have galaxywide colors of $W 1-W 2>0.5$. Thus, a ULX origin for the observed X-ray emission, given the observed X-ray luminosities and mid-infrared colors of the nuclear regions, is highly unlikely. 

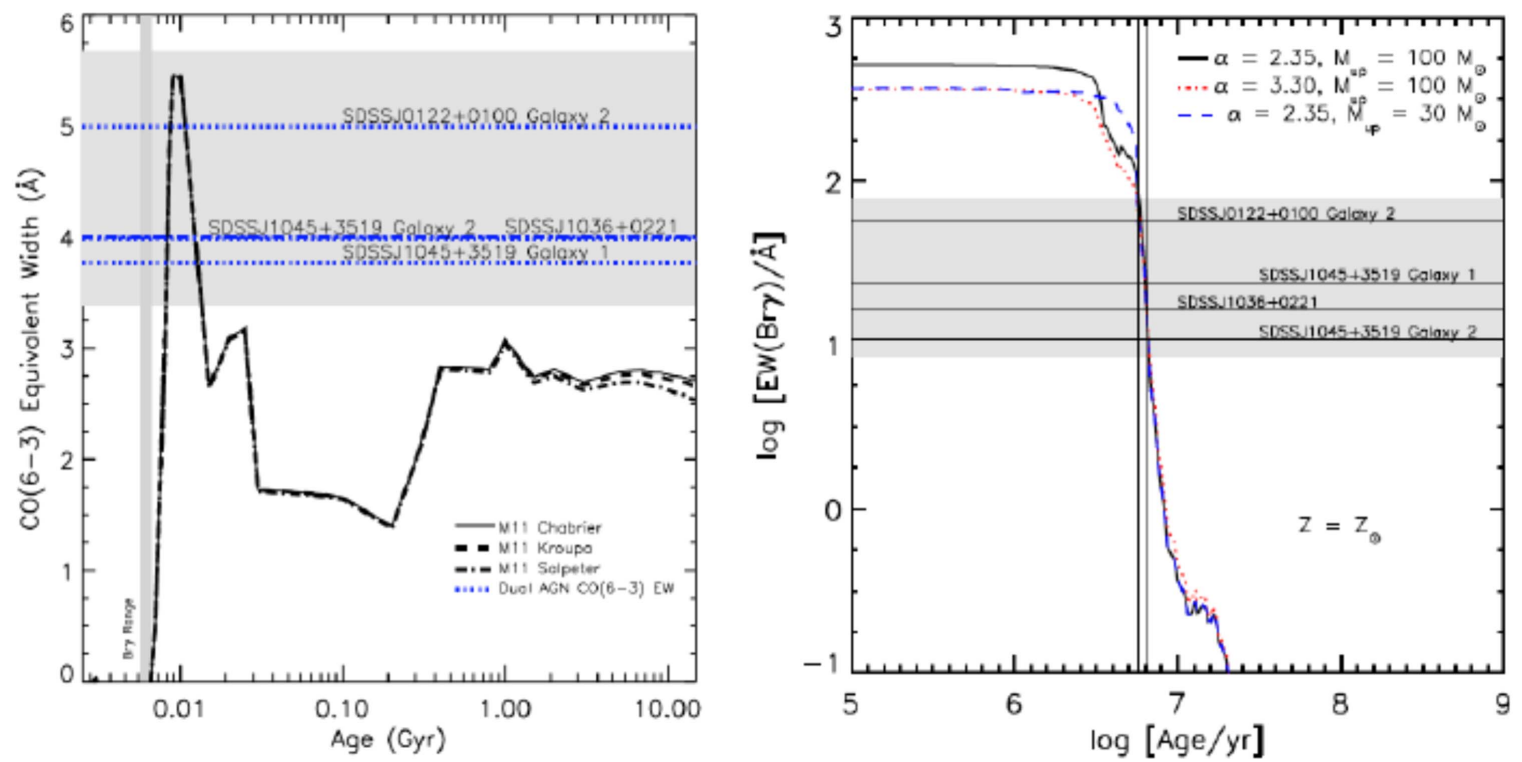

Figure 7. Left: CO EW versus age from M11 models with three different initial mass functions (Kroupa, Chabrier, Salpeter), at solar metallicity. The horizontal blue dotted lines are the measured EW of $\mathrm{CO}(6-3)$ and the gray area denotes the range of $1 \sigma$ error bars associated with these values. These measurements imply a stellar population age $<20 \mathrm{Myr}$. The vertical gray band indicates the range of ages constrained by the Br $\gamma$ EWs. Right: Br $\gamma$ EW versus age from Starburst 99 star formation models for three choices of the initial mass function, expressed in terms of the power-law exponent $\alpha$ and the upper mass cutoff $M_{\text {up }}$, at solar metallicity. The horizontal lines are the measured $\mathrm{EW}$ of $\mathrm{Br} \gamma$ and the gray area indicates the $1 \sigma$ error range associated with these measurements. The vertical lines show the age ranges constrained by the $\mathrm{Br} \gamma \mathrm{EWs}$, which is $\sim 8 \mathrm{Myr}$.

\subsection{Near-infrared AGN Diagnostics}

There are several potential diagnostics that can be used to find AGNs from our near-infrared observations. In particular, the near-infrared spectral region offers access to several collisionally excited forbidden transitions from highly ionized species, which cannot be produced by stellar processes, and since the extinction in the $\mathrm{K}$ band is roughly a factor of 10 less than that in the optical, near-infrared spectroscopy can potentially reveal hidden broad line regions (BLRs). We consider the detection of either a broad recombination line or a coronal line as confirmation of an AGN. However, the absence of a coronal line does not necessarily imply the absence of an AGN. Indeed, this line is frequently not detected even in optically confirmed Type 2 AGNs (e.g., Riffel et al. 2006; Mason et al. 2015). Even among a subsample of the Swift/BAT AGNs from the 70 month catalog, only $\approx 20 \%$ have detections in the [Si VI] line in recent follow-up observations (Lamperti et al. 2017). Similarly, the absence of a broad recombination line does not necessarily imply the absence of an AGN. Even in the Swift/BAT sample, only $10 \%$ of the optically classified Seyfert 2 galaxies show evidence for broad lines in the nearinfrared (Lamperti et al. 2017). If the extinction toward the AGNs in the targets studied here is very high, as expected for late stage mergers (L. Blecha et al. 2017, in preparation), the absence of both coronal lines and broad recombination lines should be expected. Finally, the [Fe II] $1.257 \mu \mathrm{m} / \mathrm{Pa} \beta$ and the $\mathrm{H}_{2}$ 1-0 $\mathrm{S}(1) / \mathrm{Br} \gamma$ ratio, is also a diagnostic that has been used in the literature to reveal optically obscured AGNs (Larkin et al. 1998; Rodríguez-Ardila et al. 2005; Riffel et al. 2013); however, the interpretation of the emission lines ratios from these low-ionization species is ambiguous (see Smith et al. 2014). If these line ratios are consistent with AGNs, we consider this suggestive of the presence of an AGN but not confirmation of its existence.

We detected the $[\mathrm{Si} \mathrm{VI}]$ coronal line emission in four nuclear sources, confirming the presence of AGNs at these locations. We did not detect broad near-infrared recombination lines in any of our targets. However, there is some evidence for broad wings in the $\mathrm{Pa} \alpha$ line in many of the targets, possibly indicating outflowing gas or a hidden BLR. The [Fe II] $1.257 \mu \mathrm{m} / \mathrm{Pa} \beta$ and the $\mathrm{H}_{2} 1-0 \mathrm{~S}$ (1) $/ \mathrm{Br} \gamma$ ratios are consistent with AGNs in all seven of the spectra in which all lines were measured. The details of the near-infrared spectra for these and a larger sample of mergers is presented in our future paper (see A. Constantin et al. 2017, in preparation).

In Table 7, we summarize all diagnostics used in this paper for all targets. For each source, we list the mid-infrared classification of the combined nuclei assuming the stringent three-band color cut from Jarrett et al. (2011). This midinfrared color selection uses the first 3 WISE bands to define an "AGN" region in $W 1-W 2$ versus $W 2-W 3$ color-color space that separates AGNs that dominate over their host galaxies from normal galaxies. This color cut is shown to be extremely reliable at finding luminous AGNs that dominate over the host galaxy (Mateos et al. 2012; Stern et al. 2012) at the expense of completeness. Indeed, the vast majority of optically identified AGNs in the SDSS survey are not selected using this stringent color cut (Yan et al. 2013). We list a summary classification for all targets in the last column of Table 7. We conservatively assume here that a robust identification as an AGN requires either (1) a mid-infrared color that meets the stringent three-band color cut from Jarrett et al. (2011), or (2) a $>3 \sigma$ X-ray detection with luminosity, uncorrected for intrinsic absorption in excess of $L_{2-10 \mathrm{keV}}>10^{42} \mathrm{erg} \mathrm{s}^{-1}$, or (3) the detection of a near-infrared coronal line. Based on these criteria, we report a robust detection of at least one AGN in four out of the six mergers and dual AGN candidates in four out of the six mergers. We note, however, that the observed X-ray luminosities of several duals reported in the literature are comparable to those reported in this work. Indeed, the well-studied dual AGN NGC 6240 has an intrinsic luminosity, corrected for intrinsic absorption, of $L_{2-10 \mathrm{keV}}=7 \times 10^{41} \mathrm{erg} \mathrm{s}^{-1}$ based on Chandra observations, 
Table 7

Summary of AGN Diagnostics for Each Source

\begin{tabular}{|c|c|c|c|c|c|c|}
\hline $\begin{array}{l}\text { Name } \\
\text { (SDSS) }\end{array}$ & $\log \left(L_{2-10 \mathrm{keV}}^{\text {Obs. }}\right)-\log \left(L_{2-10 \mathrm{keV}}^{\mathrm{SF}}\right)$ & $\begin{array}{l}\text { Near-IR } \\
\text { Line Ratios }\end{array}$ & $\begin{array}{l}\text { Coronal } \\
\text { Lines }\end{array}$ & $\begin{array}{l}\text { BPT } \\
\text { Class }\end{array}$ & $\begin{array}{l}\text { MIR } \\
\text { AGN }\end{array}$ & $\begin{array}{l}\text { Summary } \\
\text { Classification }\end{array}$ \\
\hline SDSS J0122+0100 & & & & & $\mathrm{Y}$ & Dual AGN Candidate \\
\hline Galaxy 1 & $0.87 \pm 0.37$ & Possible AGN & Y & SF & & \\
\hline Galaxy 2 & $1.19 \pm 0.40$ & Possible AGN/SF & $\mathrm{N}$ & SF & & \\
\hline SDSS J1036+0221 & $2.87 \pm 0.35$ & Possible AGN & $\mathrm{Y}$ & Comp. & $\mathrm{Y}$ & Single AGN \\
\hline SDSS J1045+3519 & & & & & $\mathrm{N}$ & Dual AGN Candidate \\
\hline Galaxy 1 & $1.45 \pm 0.38$ & Possible AGN & $\mathrm{N}$ & Comp & & \\
\hline Galaxy 2 & $2.87 \pm 0.45$ & Possible AGN & $\mathrm{N}$ & SF & & \\
\hline SDSS J1126+1913 & $0.37 \pm 0.40$ & $\cdots$ & $\mathrm{Y}$ & Comp. & $\mathrm{Y}$ & Single AGN \\
\hline SDSS JJ1221+1137 & & & & & $\mathrm{N}$ & Dual AGN Candidate \\
\hline Galaxy 1 & $1.03 \pm 0.36$ & Possible AGN & $\mathrm{N}$ & SF & & \\
\hline Galaxy 2 & $0.92 \pm 0.40$ & Possible AGN & $\mathrm{Y}$ & $\ldots$ & & \\
\hline SDSS J1306+0735 & & & & & $\mathrm{N}$ & Dual AGN Candidate \\
\hline Galaxy 1 & $0.46 \pm 0.36$ & $\ldots$ & $\ldots$ & SF & & \\
\hline Galaxy 2 & $2.83 \pm 0.39$ & $\cdots$ & $\cdots$ & $\ldots$ & & \\
\hline
\end{tabular}



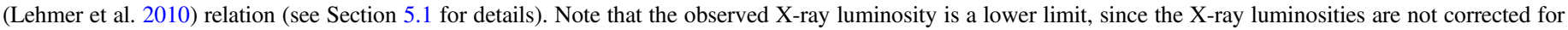

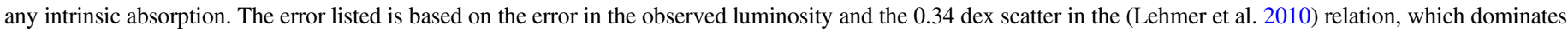

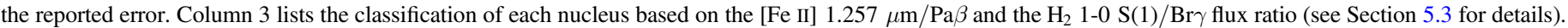

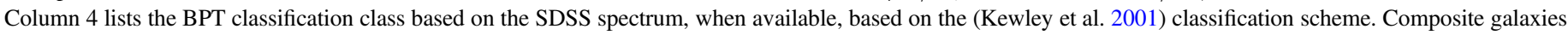

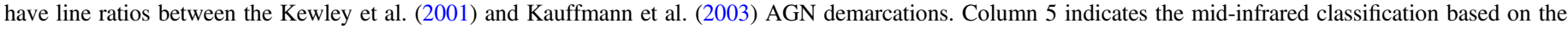

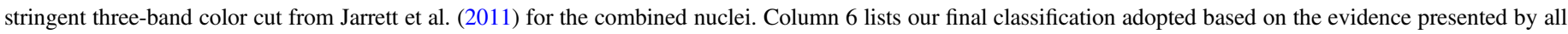
of the diagnostics used in this work.

and meets neither the $W 1-W 2>0.8$ or the Jarrett et al. (2011) color cuts.

\section{Notes on Individual Objects}

Below we discuss the individual natures of the X-ray sources for each merger summarized in Table 7.

\subsection{SDSS J0122+0100: Tentative Dual AGNs}

The northwest Chandra source of SDSS J0122+0100 (Galaxy 1) and the south east Chandra source (Galaxy 2) are detected in the full band, both with luminosities significantly above that expected from XRBs, taking into account the 0.34 dex scatter in the Lehmer et al. (2010) relation (see Table 6) indicating tentative evidence for a dual AGN system in this merger. Both galaxies show a blue wing in the $\mathrm{Pa} \alpha$ line but no detectable wing on the $\mathrm{Br} \gamma$ line. Galaxy 1 is classified as an AGN based on the [Fe II] $1.257 \mu \mathrm{m} / \mathrm{Pa} \beta$ and the $\mathrm{H}_{2}$ 1-0 $\mathrm{S}(1) / \mathrm{Br} \gamma$ ratio, a diagnostic that has been used in the literature to reveal optically obscured AGNs (Larkin et al. 1998; Rodríguez-Ardila et al. 2005; Riffel et al. 2013), although the interpretation of the emission lines ratios from these low ionization species is ambiguous (see Smith et al. 2014). Galaxy 2 has [Fe II] $1.257 \mu \mathrm{m} / \mathrm{Pa} \beta$ and $\mathrm{H}_{2}$ $1-0 \mathrm{~S}(1) / \mathrm{Br} \gamma$ ratios at the border between starbursts and AGNs and no coronal lines were detected in this source. However, the observed X-ray luminosity, uncorrected for any intrinsic absorption, is a factor of $\approx 15$ more than that expected from XRBs in this nuclei, making it unlikely that star formation alone can account for the combined properties of this source. There is a $4 \sigma$ detection of the [Si VI] $1.964 \mu \mathrm{m}$ line in Galaxy 1 that provides convincing evidence for an AGN in this nucleus. The details of the near-infrared observations are presented in A. Constantin et al. (2017, in preparation). There are two SDSS spectra for this merger, matching well the LBT positions. The optical line ratios for both spectra are in the star-forming region of the BPT diagram based on the Kewley et al. (2001) classification scheme. The combined X-ray, near-infrared spectral and midinfrared continuum properties point to possible optically hidden dual AGNs in this merger.

\subsection{SDSS J1036+0221: Single AGN}

This merger shows a firm detection of a single X-ray source with an observed luminosity, uncorrected for any instrinsic absorption, that is almost three orders of magnitude greater than that expected from XRBs. We also detect the source in the hard band. The nuclear source is also detected in the hard band, providing additional support for an AGN in this nucleus. Using the Bayesian Estimation of Hardness Ratios code (Park et al. 2006), the hardness ratio (HR) of this source, defined as $(\mathrm{H}-\mathrm{S}) /(\mathrm{H}+\mathrm{S})$ where $\mathrm{H}, \mathrm{S}=2-8,0.3-2 \mathrm{keV}$ counts, is -0.11 . Assuming a simple absorbed power-law X-ray spectrum with $\Gamma=1.8$, this corresponds to $N_{\mathrm{H}} \sim 7 \times 10^{21} \mathrm{~cm}^{-2}$. However, the single absorbed power-law model employed is most likely too simplistic, but there are insufficient counts to test more realistic multi-component spectral models. Given that $\Gamma$ ranges from about 1.4 to 2.6 in AGNs and that additional soft emission may be found in the form of scattered AGN X-ray photons and collisionally ionized diffuse gas emission, this value of $N_{\mathrm{H}}$ is therefore highly uncertain. The near-infrared spectrum at the location of the X-ray sources shows strong red and blue wings in the $\mathrm{Pa} \alpha$ line but the signal to noise ratio of the spectrum is insufficient to discern any broad wings on the $\mathrm{Br} \gamma$ line. The [Fe II] $1.257 \mu \mathrm{m} / \mathrm{Pa} \beta$ and the $\mathrm{H}_{2} 1-0 \mathrm{~S}(1) / \mathrm{Br} \gamma$ line ratios are the highest of any of the galaxies presented in this work, consistent with an AGN (A. Constantin et al. 2017, in preparation). There is $\mathrm{a}>4 \sigma$ detection of the [Si X] $1.430 \mu \mathrm{m}$ line in this source providing additional support for an AGN in this nucleus. There 
is one SDSS optical spectrum of this merger that matches the position of the LBT extraction. The optical line ratios are in the composite region of the BPT diagram according to the Kewley et al. (2001) classification scheme. Based on the combined X-ray, near-infrared spectral and mid-infrared continuum properties, there is strong evidence for a single AGN in this advanced merger.

\subsection{SDSS J1045+3519: Tentative Dual AGNs}

The western Chandra source of SDSS J1045+3519 (Galaxy 1) and the eastern Chandra source (Galaxy 2) are detected in the full band. Gal 1 is detected in the hard band. The observed luminosities, uncorrected for intrinsic absorption, are a factor of $\approx 30$ larger than that expected from XRBs in Galaxy 1 and almost three orders of magnitude larger for Galaxy 2 (see Table 6), suggesting that it is unlikely that star formation alone can account for the detections of either source in this merger. Both galaxies show [Fe II] $1.257 \mu \mathrm{m} / \mathrm{Pa} \beta$ and $\mathrm{H}_{2} \quad 1-0 \mathrm{~S}(1) / \mathrm{Br} \gamma$ line ratios well within the AGN range and there is tentative evidence for faint wings in the $\mathrm{Pa} \alpha$ line, but no coronal lines were detected (A. Constantin et al. 2017, in preparation). Based on the SDSS spectra, Galaxy 1 is classified as a composite galaxy and Galaxy 2 is classified as a star-forming galaxy according to the Kewley et al. (2001) classification scheme. Based on the combined X-ray, near-infrared spectral, and mid-infrared continuum properties, there is tentative evidence for optically hidden dual AGNs in this merger.

\subsection{SDSS J1126+1913: Single AGN}

The northeastern nucleus of this merger shows a Chandra detection in the full band, with an uncorrected luminosity that is $\approx 2$ larger than that expected from XRBs, providing tentative support for an AGN in this nucleus. There is noticeable red wing in the $\mathrm{Pa} \alpha$ line, and an $\approx 8 \sigma$ detection of the [Si VI] $1.964 \mu \mathrm{m}$ line. There is an SDSS spectrum at the location of this source consistent with a composite spectrum as can be seen in Figure 1. Given the detection of a coronal line in this nuclei, there is strong evidence for an AGN in this merger.

\subsection{SDSS JJ1221+1137: Tentative Dual AGNs}

The northeast Chandra source of SDSS JJ1221+1137 (Galaxy 1) and the southwest Chandra source (Galaxy 2) are both detected in the full band, both with luminosities in excess of that expected from XRBs, taking into account the 0.34 dex scatter in the Lehmer et al. (2010) relation (see Table 6) indicating tentative evidence for a dual AGN system in this merger. Gal 1 also shows a detection in the hard band, providing additional support for an AGN origin for the X-ray emission. Both galaxies are classified as an AGN based on the [Fe II] $1.257 \mu \mathrm{m} / \mathrm{Pa} \beta$ and the $\mathrm{H}_{2} 1-0 \mathrm{~S}(1) / \mathrm{Br} \gamma$ ratio, and Gal 2 shows a tentative $(2 \sigma)$ detection of the [Si VI] $1.964 \mu \mathrm{m}$ line (A. Constantin et al. 2017, in preparation). The SDSS spectrum of Gal 1 is consistent with a star-forming galaxy based on the (Kewley et al. 2001) classification scheme. The combined X-ray, near-infrared, and mid-infrared observations of this merger provide tentative support for dual AGNs.

\subsection{SDSS J1306+0735: Tentative Dual AGNs}

The southwestern Chandra source of (Galaxy 2) is detected, with most of the counts in the hard band. The uncorrected
$\mathrm{X}$-ray luminosity is almost a factor of 700 times greater than the luminosity expected from XRBs, providing strong support for an AGN in this nucleus. Galaxy 1 shows also shows a detection in the full band, with an uncorrected X-ray luminosity approximately three times larger than that expected from XRBs. The near-infrared spectrum is significantly affected by atmospheric absorption and no coronal lines were detected in either nucleus. There is an SDSS spectrum associated with Galaxy 1 that is classified as a star-forming galaxy (Figure 1).

\section{Discussion}

The detection of nuclear X-ray point sources by Chandra in all of the six advanced mergers presented in this work demonstrates that mid-infrared color selection is a successful pre-selection strategy for finding nuclear X-ray sources in mergers. The combined X-ray, near-infrared, and mid-infrared properties of these mergers strongly suggest that all mergers host at least one AGN, with four of the mergers showing tentative evidence for hosting dual AGNs with separations $<10 \mathrm{kpc}$, despite showing no firm evidence for AGNs based on optical spectroscopic studies. Our results demonstrate that optical studies miss a significant fraction of single and dual AGNs in advanced mergers, and that WISE pre-selection is potentially extremely effective in identifying these objects. The results presented in this work are consistent with other recent observations suggesting that AGNs in advanced mergers are likely obscured by significant gas and dust, resulting in traditional optical diagnostics failing to identify them, and suggesting that mid-infrared color selection is an effective tool in uncovering them. In our mid-infrared study of a large sample of galaxy pairs, we found that the fraction of obscured AGNs, selected using mid-infrared color selection, increases with the merger stage relative to a rigorously matched control sample, with the most energetically dominant optically obscured AGNs becoming more prevalent in the most advanced mergers (Satyapal et al. 2014; Ellison et al. 2015), where SFRs are highest (Ellison et al. 2016). A growing number of recent observational studies are also consistent with this scenario. For example, there is evidence from X-ray spectral analysis that there is an increase in the fraction of mergers in AGNs that are heavily absorbed or Compton-thick at moderate and high redshifts (Kocevski et al. 2015; Lanzuisi et al. 2015; Del Moro et al. 2016; Koss et al. 2016). In a recent hard X-ray spectral study of 52 local infrared luminous and ultraluminous galaxies, Ricci et al. (2017) found that the fraction of Compton-thick AGNs in late-stage mergers is higher than that local hard-X-ray-selected AGNs, and the absorbing column densities are maximum when the projected separation between the two nuclei are $\approx 0.4-10.8 \mathrm{kpc}$.

These observations are consistent with hydrodynamical merger simulations that predict that the most obscured phase coincides with peak SMBH growth during late stage mergers when tidal forces are the greatest, and where mid-infrared color selection is optimized to select the AGNs (L. Blecha et al. 2017, in preparation). Moreover, this is the stage where dual AGNs with pair separations $<0 \mathrm{kpc}$ are expected to be found. Using GADGET-3 (Springel \& Hernquist 2003; Springel 2005) hydrodynamic simulations processed with the SUNRISE (Jonsson 2006; Jonsson et al. 2010) radiative transfer code to model the infrared SED, L. Blecha et al. (2017, in preparation) calculate the efficacy of WISE mid-infrared color selection throughout the merger. They find that when moderate-to-high luminosity AGNs $\left(L_{\mathrm{AGN}}>10^{44} \mathrm{erg} \mathrm{s}^{-1}\right)$ are triggered in advanced major mergers, $>75 \%-80 \%$ of the AGN would 
Table 8

Compilation of Confirmed Dual AGNs

\begin{tabular}{|c|c|c|c|c|c|c|}
\hline Name & Redshift & $\begin{array}{l}\text { Separation } \\
\quad(\mathrm{kpc})\end{array}$ & $\begin{array}{l}\text { Double } \\
\text { Peaked }\end{array}$ & $\begin{array}{l}\text { Confirmation } \\
\text { Method }\end{array}$ & $\begin{array}{c}W 1-W 2 \\
\text { mag }\end{array}$ & $\begin{array}{c}\text { Reference } \\
\text { Number }\end{array}$ \\
\hline Radio Galaxy $0402+379$ & 0.055 & 0.007 & $\cdots$ & VLBA & 0.1 & 16 \\
\hline SDSS J1158+3231 & 0.166 & 0.62 & $\mathrm{Y}$ & Optical and radio & 0.5 & 14 \\
\hline SDSS J1323-0159 & 0.350 & 0.8 & & Optical & 1.3 & 18 \\
\hline NGC 6240 & 0.024 & 0.9 & $\cdots$ & Chandra and VLBI & 0.7 & 8 \\
\hline SDSS J1023+3243 & 0.127 & 1.02 & $\mathrm{Y}$ & Optical and VLA & 0.4 & 14 \\
\hline SDSS J1623+0808 & 0.199 & 1.55 & $\mathrm{Y}$ & Optical and VLA & 0.9 & 14 \\
\hline SDSS J1715+6008 & 0.157 & 1.9 & $\mathrm{Y}$ & SDSS and Chandra & 0.4 & 2 \\
\hline SDSS J1108+0659 & 0.182 & 2.1 & $\mathrm{Y}$ & Optical and Chandra & 0.7 & 11 \\
\hline SDSS J1126+2944 & 0.102 & 2.2 & $\mathrm{Y}$ & Chandra and SDSS & 0.2 & 3 \\
\hline SDSS J1425+3231 & 0.478 & 2.6 & $\mathrm{Y}$ & VLBI & 1.1 & 4 \\
\hline Mrk 739 & 0.029 & 3.4 & $\cdots$ & Chandra & 0.8 & 9 \\
\hline Mrk 463 & 0.050 & 3.8 & $\cdots$ & Chandra & 1.7 & 1 \\
\hline SDSS J2206+0003 & 0.047 & 4.1 & $\ldots$ & Optical and radio & 0.1 & 6 \\
\hline SDSS J0038+4128 & 0.073 & 4.7 & $\cdots$ & Optical & 0.6 & 7 \\
\hline SDSS J0952+2552 & 0.339 & 4.8 & $\mathrm{Y}$ & Keck AO & 1.0 & 13 \\
\hline Mrk 266 & 0.028 & 6.0 & $\cdots$ & Chandra & 0.6 & 12 \\
\hline SDSS J1146+5110 & 0.130 & 6.3 & $\mathrm{Y}$ & Optical and Chandra & 0.6 & 11 \\
\hline $3 \mathrm{C} 75 \mathrm{~N}$ & 0.023 & 6.4 & $\ldots$ & VLA & 0.0 & 15 \\
\hline $3 \mathrm{C} 75 \mathrm{~S}$ & 0.023 & 6.4 & $\cdots$ & VLA & -0.1 & 15 \\
\hline SDSS J0051+0020 & 0.113 & 7.1 & $\cdots$ & Optical and radio & 0.5 & 6 \\
\hline SDSS J1502+1115 & 0.391 & 7.4 & $\mathrm{Y}$ & EVLA and optical & 1.5 & 5 \\
\hline SDSS J2300-0005 & 0.180 & 7.7 & $\cdots$ & Optical and radio & 0.3 & 6 \\
\hline IRAS $05589+2828$ & 0.033 & 8.0 & $\cdots$ & Chandra and BAT & 1.0 & 10 \\
\hline Was 49a & 0.06 & 8.3 & $\cdots$ & Optical & 0.1 & 19 \\
\hline Was $49 b$ & 0.06 & 8.3 & $\cdots$ & Optical & 1.3 & 19 \\
\hline SDSS J1407+4428 & 0.143 & 8.3 & $\cdots$ & Chandra and Optical & 0.8 & 20 \\
\hline SDSS J1107+6506N & 0.033 & 8.8 & $\ldots$ & Chandra and SDSS & 0.0 & 17 \\
\hline SDSS J1107+6506S & 0.033 & 8.8 & $\cdots$ & Chandra and SDSS & 0.1 & 17 \\
\hline SDSS J2232+0012 & 0.221 & 11.6 & $\cdots$ & Optical and radio & 0.7 & 6 \\
\hline
\end{tabular}



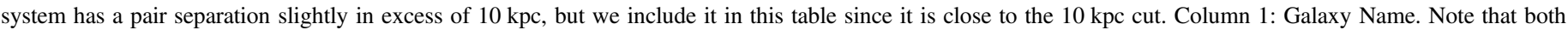

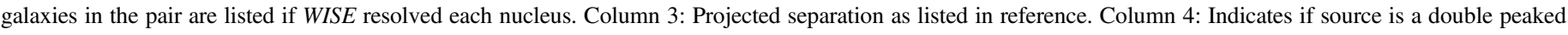

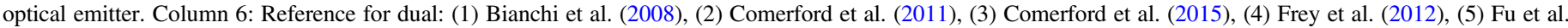

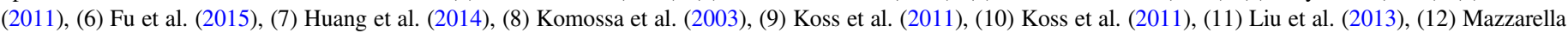

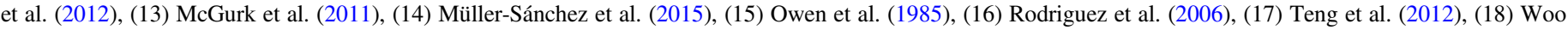
et al. (2014), (19) Bothun et al. (1989), Moran et al. (1992), Secrest et al. (2017), (20) Ellison et al. (2017).

typically be identified with a WISE color cut of $W 1-W 2>0.5$. Also, at these luminosities, dual AGN systems would be identified via this criterion with nearly $100 \%$ efficiency.

The high success rate of mid-infrared color selection in finding dual AGN candidates demonstrated in this work is therefore completely consistent with simulations as shown by L. Blecha et al. (2017, in preparation). Indeed, a significant fraction of all known duals with separations $\lesssim 10 \mathrm{kpc}$ in the literature, many of which were discovered through serendipitous X-ray observations, have red mid-infrared colors. In a complementary study, Ellison et al. (2017) used a combination of MaNGA IFU spectroscopy and Chandra to identify another dual AGN in a late stage merger with $8 \mathrm{kpc}$ separation. In Table 8, we list a compilation of known duals with separations $\lesssim 10 \mathrm{kpc}$ reported in the literature. We selected sources that are listed as confirmed by the authors, and list the confirmation method employed in the reference, which includes X-ray, radio, spatially resolved optical spectroscopy on both nuclei in the pair, and a combination of these methods. The SDSS $r$-band images of these confirmed duals is shown in Figure 8, ordered by increasing physical pair separation. In Figure 9 we show the WISE color-color diagram showing the colors of the confirmed duals listed in Table 8, as well as our targets. As can be seen, $\approx 62 \%$ of all confirmed dual AGNs in the literature have $W 1-W 2>0.5$. We also show the more stringent three-band color cut from Jarrett et al. (2011), in which $\approx 1 / 3$ of the duals reside. According to the simulations from L. Blecha et al. (2017, in preparation), the $W 1-W 2$ color increases with merger stage both because of an increasing SFR and an increase in the accretion rate onto the SMBH. For pair separations $<10 \mathrm{kpc}$, before the peak in the SMBH accretion rate, the WISE color rises above $W 1-W 2>0.5$ with an enhancement in the star formation activity when both SMBHs are also accreting, consistent with observations (Ellison et al. 2016). Thus, while star formation alone can in principle generate extreme mid-infrared colors, the less stringent color cut of $W 1-W 2>0.5$ selects a merger state that has a high probability of also being a dual AGN candidate, as this work has demonstrated. Thus, the less restrictive color cut of $W 1-W 2>0.5$ employed in this work is a more effective pre-selection strategy for finding dual candidates in advanced mergers. Indeed, Blecha et al. show that the more stringent color-cut of $W 1-W 2>0.8$ actually misses a significant fraction of dual AGNs. For the closest pair separations up until coaelescence, the bolometric luminosity of the AGN is expected to be greatest (Ellison et al. 2011; Satyapal 


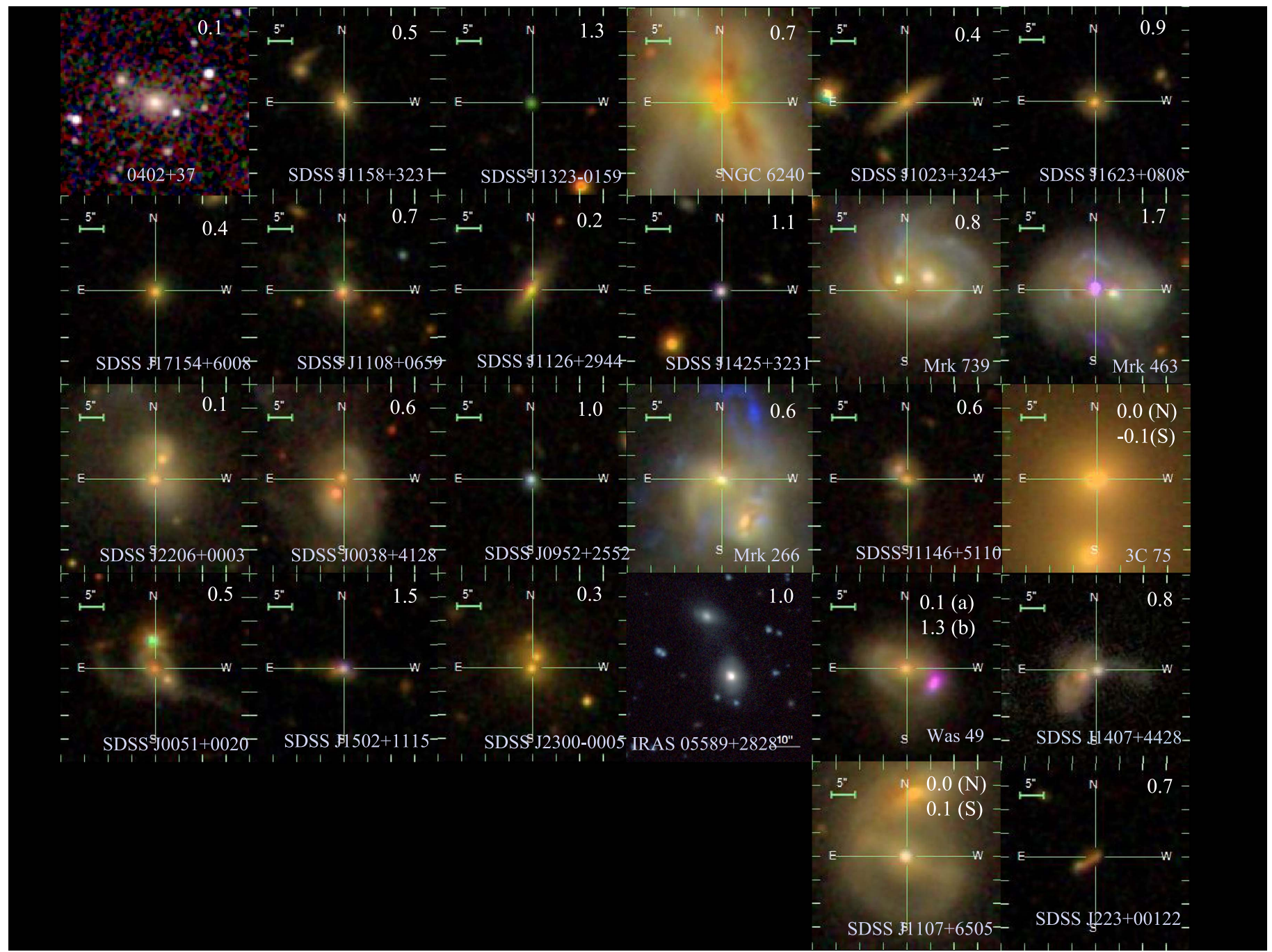

Figure 8. SDSS $r$-band images (when available) of confirmed duals with separations $\lesssim 10 \mathrm{kpc}$ from the literature (listed in Table 8 ) ordered by increasing physical pair separation. The $W 1-W 2$ color for each source is indicated in the upper right corner. Note that for IRAS $05589+282$, we show the three-color JHK UKIDSS image, and for Radio Galaxy $0402+379$, we show the three-color JHK 2MASS image.

et al. 2014), resulting in the reddest $W 1-W 2$ colors. At this stage, the AGN dominates the bolometric luminosity, resulting in AGNs that would be identified through the more stringent three-band Jarrett et al. (2011) demarcation. While there are only a few known dual AGNs with separations $<10 \mathrm{kpc}$, the Jarrett et al. (2011) AGNs identified in Figure 9 are the most luminous known duals as can be seen from Figure 10. These findings are consistent with the results from the Swift/BAT survey by Koss et al. (2012), who find that the luminosities of dual AGNs increase with decreasing pair separations (see also Ellison et al. 2017).

Based on the simulations from L. Blecha et al. (2017, in preparation), the gas column densities toward the SMBHs are predicted to be high for pair separations $<10 \mathrm{kpc}$, peaking just prior to coalescence, with declining but significant obscuration persisting 50-100 Myr post-merger as AGN feedback drives the gas outward, lowering the column densities. The predicted column densities for pair separations $<10 \mathrm{kpc}$ are expected to significantly lower the observed X-ray luminosity relative to the mid-infrared luminosity, consistent with our results. The midinfrared luminosity, thought to be re-emitted by the obscuring torus, and the AGN intrinsic $2-10 \mathrm{keV}$, are known to follow a tight correlation over several orders of magnitude (Lutz et al.
2004; Gandhi et al. 2009; Mateos et al. 2015). In Figure 11, we plot the $12 \mu \mathrm{m}$ luminosity, calculated by interpolating the $W 2$ and $W 3$ band luminosities, versus the observed hard X-ray luminosity for the advanced mergers in our sample, together with the sample of hard-X-ray-selected AGNs from the 70 month Swift/BAT survey (Ricci et al. 2015) for which a detailed broadband spectral analysis enables a direct determination of the intrinsic absorption, showing unabsorbed $\left(N_{\mathrm{H}}<10^{22} \mathrm{~cm}^{-2}\right)$, absorbed $\left(N_{\mathrm{H}}=10^{22-24} \mathrm{~cm}^{-2}\right)$, and Compton-thick $\left(N_{\mathrm{H}}>\right.$ $10^{24} \mathrm{~cm}^{-2}$ ) AGNs. The mergers (based on visual inspection of the optical images, where available) in the Swift/BAT sample have been excluded from the plot, since we are interested in comparing the advanced mergers in this work, with isolated hard-X-ray-selected AGNs. We also exclude blazars from the Swift/BAT catalog, since the IR and X-ray emission are produced in different regions with respect to non-blazar AGNs. We also plot in Figure 11, the effect of absorption on the unabsorbed best fit linear relation from the Swift/BAT sample (dashed line). The dotted lines correspond to different intrinsic $\left(N_{\mathrm{H}}\right)$ values and were calculated using the MYTORUS model (Murphy \& Yaqoob 2009), which self-consistently considers absorption and reprocessed X-ray radiation from a toroidal absorber. MYTorus is distributed in three tables that take into 


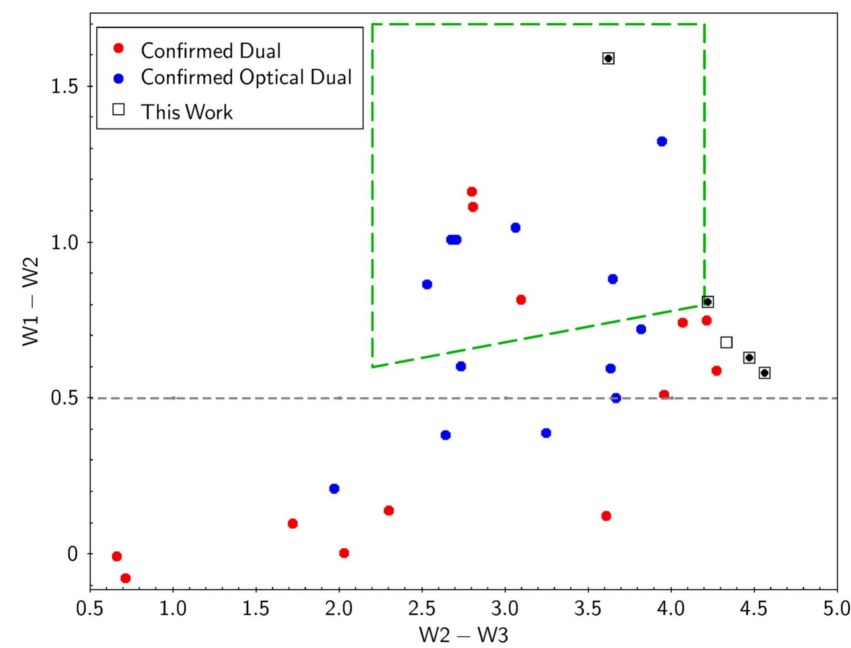

Figure 9. WISE color-color diagram showing the colors of the confirmed duals compiled from the literature listed in Table 8 , with those with both nuclei identified optically denoted by the blue circles. Note that if the nuclei are resolved by WISE, the color of each nucleus is plotted. Otherwise, the color represents the combined color of both nuclei. We also plot the WISE colors of the advanced mergers from this work shown by the black open squares, with the dual candidates indicated with squares with a central black circle. The three-band color cut from Jarrett et al. (2011) is displayed by the dotted wedge, and a color cut of $W 1-W 2>0.5$ is indicated by the dashed horizontal line.

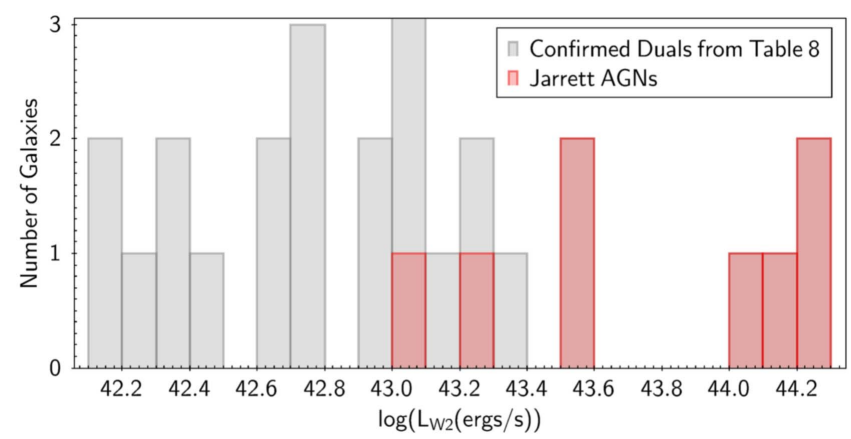

Figure 10. Histogram showing the $W 2$ luminosities of the confirmed duals compiled from the literature listed in Table 8 . Mergers meeting the three-band color cut from Jarrett et al. (2011) are identified in blue.

account the absorbed primary X-ray emission (MYTORUSZ), the scattered component (MYTORUSS), and the fluorescent lines (MYTORUSL). For the X-ray continuum we used a power law with a photon index of $\Gamma=1.8$, and fixed the inclination angle with respect to the symmetry axis of the system to $\theta_{i}=90^{\circ}$. In XSPEC, the model used is a combination of the three components of the MYTorus model: (MYTorusZ $\times$ zpowerlaw + MYTorusS + MYTorusL). As can be seen, the observed $\mathrm{X}$-ray-to-mid-infrared flux ratios in all of the advanced mergers are low compared to optically identified and hard-X-ray-selected AGNs, comparable to the most obscured sources in the Swift/ BAT survey, and several of the confirmed duals listed in Table 8, suggesting heavy obscuration corresponding to intrinsic absorption $N_{\mathrm{H}}$ of a few times $10^{24} \mathrm{~cm}^{-2}$, precisely as predicted by the simulations from L. Blecha et al. (2017, in preparation). The X-ray to midinfrared flux ratios of our sample are comparable to well-studied mergers for which $N u S T A R$ and/or broadband X-ray spectral analysis using multiple facilities confirms an intrinsic absorption $N_{\mathrm{H}}$ in excess of a few times $10^{23} \mathrm{~cm}^{-2}$, such as NGC 6240, Mrk 273, and UGC 5101 (Teng et al. 2012; Corral et al. 2014; Ricci



Figure 11. The hard X-ray luminosity versus the mid-infrared luminosity for the sample of hard-X-ray-selected AGNs from the 70 month Swift/BAT survey for which a detailed broadband spectral analysis enables a direct determination of the intrinsic absorption (Ricci et al. 2015, 2016), showing unabsorbed $\left(N_{\mathrm{H}}<10^{22} \mathrm{~cm}^{-2}\right)$, absorbed $\left(N_{\mathrm{H}}=10^{22-24} \mathrm{~cm}^{-2}\right)$, and Compton-thick $\left(N_{\mathrm{H}}>10^{24} \mathrm{~cm}^{-2}\right)$ AGNs. We also plot the advanced mergers from this work, and the confirmed duals listed in Table 8 for which X-ray and mid-infrared fluxes are available. The dotted lines display the predicted relations for various column densities using a MyTorus model (see Section 7 for details).

Table 9

Intrinsic Absorption Estimates

\begin{tabular}{lccc}
\hline \hline Name & $\begin{array}{c}\log L_{2-10}^{\text {Obs. }} \\
\left(\mathrm{erg} \mathrm{s}^{-1}\right)\end{array}$ & $\begin{array}{l}L_{2-10 \mathrm{keV}}^{\text {Intr. }} \\
\left(\mathrm{erg} \mathrm{s}^{-1}\right)\end{array}$ & $10^{24}\left(\mathrm{~cm}^{-2}\right)$ \\
\hline J1036+0221 & 41.35063 & 42.8337 & $0.9_{-0.04}^{+0.07}$ \\
$\mathrm{~J} 0122+0100$ & 41.07003 & 43.4158 & $3.0_{-1.2}^{+1.6}$ \\
$\mathrm{~J} 1221+1137$ & 41.44890 & 43.6861 & $2.7_{-1.1}^{+1.5}$ \\
$\mathrm{~J} 1126+1913$ & 40.68518 & 43.4761 & $5.0_{-1.7}^{+2.2}$ \\
$\mathrm{~J} 1306+0735$ & 41.35650 & 43.5252 & $2.4_{-1.0}^{+1.4}$ \\
$\mathrm{~J} 1045+3519$ & 41.21140 & 43.5772 & $3.1_{-1.2}^{+1.7}$ \\
\hline
\end{tabular}

Note. Column 4 lists the implied intrinsic absorption $N_{\mathrm{H}}$ for our sample based on the observed X-ray to mid-infrared flux ratio using the linear regression for the unabsorbed Swift/BAT sources, assuming the MYTORUS model described in Section 7.

et al. 2015, 2016; Puccetti et al. 2016; Oda et al. 2017). In Table 9, we list the implied intrinsic absorption $N_{\mathrm{H}}$ for our sample based on the observed X-ray-to-mid-infrared flux ratio using the linear regression for the unabsorbed Swift/BAT sources. The low X-ray-to-mid-infrared fluxes of our advanced mergers in our sample are consistent with the low X-ray-to[O III] $\lambda 5007$ fluxes observed in other known dual AGN systems (Liu et al. 2013; Comerford et al. 2015), strongly suggesting that the low X-ray-to- [O III] $\lambda 5007$ flux ratios are also due to higher nuclear gas concentrations in advanced mergers.

We note that the value of $N_{\mathrm{H}}$ and hence intrinsic hard X-ray luminosities listed in Table 9 must be viewed with some caution since these values depend on the X-ray model assumed and do not include an extinction correction to the $12 \mu \mathrm{m}$ band luminosity, which may be significant for some Compton-thick sources (e.g., Goulding et al. 2012). Furthermore, we are assuming that the suppression of X-ray emission relative to the mid-infrared emission in our sources is due entirely to absorption and not intrinsic X-ray weakness, and that the contribution from star formation in the two bands is negligible. It is likely that much of the scatter in Figure 11 is likely due to contamination of the mid-infrared flux from star formation. 
Indeed, recent high spatial resolution mid-infrared observations have demonstrated that the scatter in the X-ray to mid-infrared relation of well-studied AGNs is significantly reduced when the nuclear mid-infrared fluxes are used (Asmus et al. 2015). These results are consistent with earlier studies that showed that low observed X-ray to mid-infrared luminosities alone cannot definitely ascertain whether a Compton-thick AGN is present, albeit the majority of such systems are heavily obscured (Georgantopoulos et al. 2011). In the case of our sample, which consists of mergers with active star formation, the intrinsic absorption listed in Table 9 is likely an overestimate.

\section{Summary and Conclusions}

We have presented Chandra/ACIS and XMM-Newton observations and near-infrared spectraobtained with the LBT of six advanced mergers with nuclear separations $<10 \mathrm{kpc}$ preselected through mid-infrared color selection using WISE.

Our main results can be summarized as follows:

1. We detect at least one nuclear X-ray source in all six mergers, with four sources showing detections in the hard band. We report detections of a secondary source in four out of the six mergers. Note that the lack of a detection in the two mergers with a single $\mathrm{X}$-ray source does not exclude the possibility of a fainter secondary source, below our detection threshold.

2. The observed X-ray luminosity in all targets is significantly above that expected from star formation in the host galaxy. The detection of near-infrared coronal lines, together with near-infrared line diagnostics typically associated with AGNs, and the mid-infrared colors of all mergers strongly suggests the presence of buried AGNs in four out of the six advanced mergers, with four showing possible evidence for duals AGNs with pair separations $<10 \mathrm{kpc}(\mathrm{J} 1036+0221, d=2.8 \mathrm{kpc} ; \mathrm{J} 1306$ $+0735 ; 2.0 \sigma$ in $\mathrm{J} 1221+1137 ; 1.5 \sigma$ in $\mathrm{J} 1045+3519$. None of these mergers are identified as dual AGNs through optical spectroscopy. While the data presented here are highly suggestive of dual AGNs in these four mergers, the possibility that emission from the secondary source can be produced by star formation in the host cannot be ruled out.

3. All of the advanced mergers in our sample have observed 2-10 leV X-ray luminosities that are low relative to their mid-infrared luminosities compared with local hard-Xray-selected unabsorbed AGNs, comparable to the most obscured sources in the Swift/BAT survey, and several of the other confirmed well-known duals in the literature, suggesting heavy obscuration corresponding to intrinsic absorption $N_{\mathrm{H}}$ of a few times $10^{24} \mathrm{~cm}^{-2}$. We suggest that these low X-ray to mid-infrared flux ratios are due to higher gas column densities and enhanced star formation activity contributing to the mid-infrared flux.

4. The detection of buried AGNs in advanced mergers, along with the possible success of mid-infrared color selection of $W 1-W 2>0.5$ in finding duals, is consistent with recent hydrodynamical merger simulations that show that obscured luminous AGNs should be a natural occurrence in advanced mergers, where dual AGNs are likely to be found, and that mid-infrared color-selection is the ideal way to find them.
These observations demonstrate that mid-infrared color selection, and in particular a color cut of $W 1-W 2>0.5$, is a promising pre-selection strategy for finding single and dual AGN candidates in advanced mergers. If the 4 dual AGN candidates are confirmed, the pilot study presented in this work would increase the sample of all known dual AGN candidates with pair separations $<10 \mathrm{kpc}$ by almost $15 \%$. We have applied this technique recently to an advanced merger with red midinfrared colors in the MANGA sample and confirmed the AGN (Ellison et al. 2017). Follow-up observations of a larger sample of these mergers can potentially vastly increase the sample of known dual AGNs compared with optical, and blind X-ray searches. While radio surveys do not suffer from obscuration bias, the radio emission in advanced mergers can be dominated by and indistinguishable from compact nuclear starbursts (Condon et al. 1991; Del Moro et al. 2013).

Moreover, our results, coupled with theoretical predictions, imply that a key stage in the evolution of galaxies, which contributes significantly to the SMBH accretion history of the universe, is potentially being missed by past studies.

We gratefully acknowledge the anonymous referee for a very thorough and insightful review that improved this manuscript. N.J.S. and S.S. gratefully acknowledge support by the Chandra Guest Investigator Program under NASA Grant G01-12126X. P.M. gratefully acknowledges support from a Mason 4-VA grant. A.C. and J.F. gratefully acknowledge support from a JMU 4-VA Collaborative grant. It is a pleasure to acknowledge the support of Raj Kiran Koju, for his help in assembling the initial sample of advanced mergers, without which this work would never have begun. We also gratefully acknowledge the support of Seth Mowry for his meticulous assembly of the initial compilation of confirmed duals included in this work. We are very grateful to Peter Edmonds, Ryan Hickox, and Vinay Kashyap for their insight on source significance estimates in the low count regime used in this paper.

This publication makes use of data products from the Widefield Infrared Survey Explorer, which is a joint project of the University of California, Los Angeles, and the Jet Propulsion Laboratory/California Institute of Technology, funded by the National Aeronautics and Space Administration. Funding for SDSS-III has been provided by the Alfred P. Sloan Foundation, the Participating Institutions, the National Science Foundation, and the U.S. Department of Energy Office of Science. The SDSS-III web site is http://www.sdss3.org/.

SDSS-III is managed by the Astrophysical Research Consortium for the Participating Institutions of the SDSS-III Collaboration including the University of Arizona, the Brazilian Participation Group, Brookhaven National Laboratory, Carnegie Mellon University, University of Florida, the French Participation Group, the German Participation Group, Harvard University, the Instituto de Astrofisica de Canarias, the Michigan State/Notre Dame/JINA Participation Group, Johns Hopkins University, Lawrence Berkeley National Laboratory, Max Planck Institute for Astrophysics, Max Planck Institute for Extraterrestrial Physics, New Mexico State University, New York University, Ohio State University, Pennsylvania State University, University of Portsmouth, Princeton University, the Spanish Participation Group, University of Tokyo, University of Utah, Vanderbilt University, University of Virginia, University of Washington, and Yale University. This research has made use of the NASA/IPAC Extragalactic Database (NED) which is operated by the Jet 
Propulsion Laboratory, California Institute of Technology, under contract with the National Auronautics and Space Administration. We also gratefully acknowledge the use of the software TOPCAT (Taylor 2005) and Astropy (Astropy Collaboration et al. 2013).

The LBT is an international collaboration among institutions in the United States, Italy, and Germany. LBT Corporation partners are: The University of Arizona on behalf of the Arizona Board of Regents; Istituto Nazionale di Astrofisica, Italy; LBT Beteiligungsgesellschaft, Germany, representing the Max-Planck Society, The Leibniz Institute for Astrophysics Potsdam, and Heidelberg University; The Ohio State University, and The Research Corporation, on behalf of The University of Notre Dame, University of Minnesota and University of Virginia.

This research was performed while N.J.S. held an NRC Research Associateship award at the Naval Research Laboratory. Basic research in astronomy at the Naval Research Laboratory is funded by the Office of Naval Research.

\section{ORCID iDs}

Shobita Satyapal (iD https://orcid.org/0000-0003-2277-2354 Nathan J. Secrest (iD https://orcid.org/0000-0002-4902-8077 Claudio Ricci (1D https://orcid.org/0000-0001-5231-2645 Sara L. Ellison (ib https://orcid.org/0000-0002-1768-1899 Barry Rothberg (i) https://orcid.org/0000-0003-2283-2185 Laura Blecha (i) https://orcid.org/0000-0002-2183-1087 Anca Constantin (i) https://orcid.org/0000-0002-2441-1619 Mario Gliozzi (i) https://orcid.org/0000-0002-8818-9009

\section{References}

Abbott, B. P., Abbott, R., Abbott, T. D., et al. 2016, PhRvL, 116, 061102 Arzoumanian, Z., Brazier, A., Burke-Spolaor, S., et al. 2016, ApJ, 821, 13 Asmus, D., Gandhi, P., Hönig, S. F., Smette, A., \& Duschl, W. J. 2015, MNRAS, 454, 766

Assef, R. J., Stern, D., Kochanek, C. S., et al. 2013, ApJ, 772, 26

Baldwin, J. A., Phillips, M. M., \& Terlevich, R. 1981, PASP, 93, 5

Ballo, L., Braito, V., Della Ceca, R., et al. 2004, ApJ, 600, 634

Barrows, R. S., Comerford, J. M., Greene, J. E., \& Pooley, D. 2016, ApJ, 829,37

Barrows, R. S., Comerford, J. M., Greene, J. E., \& Pooley, D. 2017, ApJ, 838, 129

Baumgartner, W. H., Tueller, J., Markwardt, C. B., et al. 2013, ApJS, 207, 19 Bianchi, S., Chiaberge, M., Piconcelli, E., Guainazzi, M., \& Matt, G. 2008, MNRAS, 386, 105

Blecha, L., Loeb, A., \& Narayan, R. 2013, MNRAS, 429, 2594

Bothun, G. D., Schmitz, M., Halpern, J. P., Lonsdale, C. J., \& Impey, C. 1989, ApJS, 70, 271

Brassington, N. J., Ponman, T. J., \& Read, A. M. 2007, MNRAS, 377, 1439

Cisternas, M., Jahnke, K., Inskip, K. J., et al. 2011, ApJ, 726, 57

Comerford, J. M., Gerke, B. F., Stern, D., et al. 2012, ApJ, 753, 42

Comerford, J. M., Pooley, D., Barrows, R. S., et al. 2015, ApJ, 806, 219

Comerford, J. M., Pooley, D., Gerke, B. F., \& Madejski, G. M. 2011, ApJL, 737, L19

Comerford, J. M., Schluns, K., Greene, J. E., \& Cool, R. J. 2013, ApJ, 777, 64

Condon, J. J., Huang, Z.-P., Yin, Q. F., \& Thuan, T. X. 1991, ApJ, 378, 65

Corral, A., Georgantopoulos, I., Watson, M. G., et al. 2014, A\&A, 569, A71 Darg, D. W., Kaviraj, S., Lintott, C. J., et al. 2010, MNRAS, 401, 1043

Del Moro, A., Alexander, D. M., Bauer, F. E., et al. 2016, MNRAS, 456, 2105 Del Moro, A., Alexander, D. M., Mullaney, J. R., et al. 2013, A\&A, 549, A59

Donley, J. L., Rieke, G. H., Pérez-González, P. G., Rigby, J. R., \& Alonso-Herrero, A. 2007, ApJ, 660, 167

Ellison, S. L., Patton, D. R., \& Hickox, R. C. 2015, MNRAS, 451, L35

Ellison, S. L., Patton, D. R., Mendel, J. T., \& Scudder, J. M. 2011, MNRAS, 418, 2043

Ellison, S. L., Secret, N. J., Mendel, J. T., Satyapal, S., \& Simard, L. 2017, MNRAS, 470, 49
Ellison, S. L., Teimoorinia, H., Rosario, D. J., \& Mendel, J. T. 2016, MNRAS, 458, L34

Fan, L., Fang, G., Chen, Y., et al. 2014, ApJL, 784, L9

Fan, L., Han, Y., Fang, G., et al. 2016, ApJL, 822, L32

Feng, H., \& Soria, R. 2011, Natur, 55, 166

Foreman, G., Volonteri, M., \& Dotti, M. 2009, ApJ, 693, 1554

Frey, S., Paragi, Z., An, T., \& Gabányi, K. É 2012, MNRAS, 425, 1185

Fu, H., Wrobel, J. M., Myers, A. D., Djorgovski, S. G., \& Yan, L. 2015, ApJL, 815, L6

Fu, H., Yan, L., Myers, A. D., et al. 2012, ApJ, 745, 67

Fu, H., Zhang, Z.-Y., Assef, R. J., et al. 2011, ApJL, 740, L44

Gabányi, K. É, Frey, S., Xiao, T., et al. 2014, MNRAS, 443, 1509

Gandhi, P., Horst, H., Smette, A., et al. 2009, A\&A, 502, 457

Gao, Y., Wang, Q. D., Appleton, P. N., \& Lucas, R. A. 2003, ApJL, 596, L171

Georgantopoulos, I., Rovilos, E., Akylas, A., et al. 2011, A\&A, 534, A23

Gilfanov, M. 2004, MNRAS, 349, 146

Goulding, A. D., Alexander, D. M., Bauer, F. E., et al. 2012, ApJ, 755, 5

Hennawi, J. F., Myers, A. D., Shen, Y., et al. 2010, ApJ, 719, 1672

Hopkins, P. F., Kocevski, D. D., \& Bundy, K. 2014, MNRAS, 445, 823

Huang, Y., Liu, X.-W., Yuan, H.-B., et al. 2014, MNRAS, 439, 2927

Ichikawa, K., Ricci, C., Ueda, Y., et al. 2017, ApJ, 835, 74

Jarrett, T. H., Cohen, M., Masci, F., et al. 2011, ApJ, 735, 112

Jonsson, P. 2006, MNRAS, 372, 2

Jonsson, P., Groves, B. A., \& Cox, T. J. 2010, MNRAS, 403, 17

Kalberla, P. M. W., Burton, W. B., Hartmann, D., et al. 2005, A\&A, 440, 775

Kauffmann, G., Heckman, T. M., Tremonti, C., et al. 2003, MNRAS, 346, 1055

Kennicutt, R. C., Jr., Tamblyn, P., \& Congdon, C. E. 1994, ApJ, 435, 22

Kewley, L. J., Dopita, M. A., Sutherland, R. S., Heisler, C. A., \& Trevena, J. 2001, ApJ, 556, 121

Kocevski, D. D., Brightman, M., Nandra, K., et al. 2015, ApJ, 814, 104

Kocevski, D. D., Faber, S. M., Mozena, M., et al. 2012, ApJ, 744, 148

Komossa, S., Burwitz, V., Hasinger, G., et al. 2003, ApJL, 582, L15

Koss, M., Mushotzky, R., Treister, E., et al. 2011, ApJL, 735, L42

Koss, M., Mushotzky, R., Treister, E., et al. 2012, ApJL, 746, L22

Koss, M. J., Assef, R., Baloković, M., et al. 2016, ApJ, 825, 85

Kriss, G. 1994, in ASP Conf. Ser. 61, Astronomical Data Analysis Software and Systems III, ed. D. R. Crabtree, R. J. Hanisch, \& J. Barnes (San Francisco, CA: ASP), 437

Lamperti, I., Koss, M., Trakhtenbrot, B., et al. 2017, MNRAS, 467, 540

Landini, M., Natta, A., Salinari, P., Oliva, E., \& Moorwood, A. F. M. 1984, A\&A, 134, 284

Lansbury, G. B., Alexander, D. M., Del Moro, A., et al. 2014, ApJ, 785, 17

Lanzuisi, G., Ranalli, P., Georgantopoulos, I., et al. 2015, A\&A, 573, A137

Larkin, J. E., Armus, L., Knop, R. A., Soifer, B. T., \& Matthews, K. 1998, ApJS, 114, 59

Lehmer, B. D., Alexander, D. M., Bauer, F. E., et al. 2010, ApJ, 724, 559

Leitherer, C., Ekström, S., Meynet, G., et al. 2014, ApJS, 212, 14

Leitherer, C., Schaerer, D., Goldader, J. D., et al. 1995, ApJS, 123, 3

Lentati, L., Shannon, R. M., Coles, W. A., et al. 2016, MNRAS, 458, 2161

Linden, T., Kalogera, V., Sepinsky, J. F., et al. 2010, ApJ, 725, 1984

Lintott, C. J., Schawinski, K., Slolsar, A., et al. 2008, MNRAS, 389, 1179

Liu, X., Civano, F., Shen, Y., et al. 2013, ApJ, 762, 110

Liu, X., Shen, Y., Strauss, M. A., \& Greene, J. E. 2010, ApJ, 708, 427

Luo, B., Brandt, W. N., Xue, Y. Q., et al. 2017, ApJS, 228, 2

Lutz, D., Maiolino, R., Spoon, H. W. W., \& Moorwood, A. F. M. 2004, A\&A, 418, 465

Mapelli, M., Moore, B., Giordano, L., et al. 2008, MNRAS, 383, 230

Maraston, C., \& Strömbäck, G. 2011, MNRAS, 418, 2785

Mason, R. E., Rodríguez-Ardila, A., Martins, L., et al. 2015, ApJS, 217, 13

Mateos, S., Alonso-Herrero, A., Carrera, F. J., et al. 2012, MNRAS, 426, 3271

Mateos, S., Carrera, F. J., Alonso-Herrero, A., et al. 2015, MNRAS, 449 1422

Mazzarella, J. M., Iwasawa, K., Vavilkin, T., et al. 2012, AJ, 144, 125

McGurk, R. C., Max, C. E., Medling, A. M., Shields, G. A., \& Comerford, J. M. 2015, ApJ, 811, 14

McGurk, R. C., Max, C. E., Rosario, D. J., et al. 2011, ApJL, 738, L2

Mechtley, M., Jahnke, K., Windhorst, R. A., et al. 2016, ApJ, 830, 156

Merritt, D., \& Milosavljević, M. 2005, LRR, 8, 8

Mihos, J., \& Hernquist, L. 1996, ApJ, 464, 641

Moran, E. C., Halpern, J. P., Bothun, G. D., \& Becker, R. H. 1992, AJ, 104,990

Müller-Sánchez, F., Comerford, J. M., Nevin, R., et al. 2015, ApJ, 813, 103

Murphy, K. D., \& Yaqoob, T. 2009, MNRAS, 397, 1549

Oda, S., Tanimoto, A., Ueda, Y., et al. 2017, ApJ, 835, 179 
Oliva, E., Origlia, L, Kotilaninen, J. K., \& Moorwood, A. F. M. 1995, A\&A, 301, 55

Origilia, L, Moorwood, A. F. M, \& Oliva, E. 1998, A\&A, 280, 536

Origlia, L., Ferraro, F. R., Fusi Pecci, F., \& Oliva, E. 1997, A\&A, 321, 859

Osterbrock, D. E., \& Ferland, G. J. 2006, Astrophysics of Gaseous Nebulae and Active Galactic Nuclei (2nd ed.; Sausalito: Univ. Science Books)

Owen, F. N., O’Dea, C. P., Inoue, M., \& Eilek, J. A. 1985, ApJL, 294, L85

Park, T., Kashyap, V. L., Siemiginowska, A., et al. 2006, ApJ, 652, 610

Puccetti, S., Comastri, A., Bauer, F. E., et al. 2016, A\&A, 585, A157

Ricci, C., Bauer, F. E., Treister, E., et al. 2016, ApJ, 819, 4

Ricci, C., Bauer, F. E., Treister, E., et al. 2017, MNRAS, 468, 1273

Ricci, C., Ueda, Y., Koss, M. J., et al. 2015, ApJL, 815, L13

Richards, G. T., Lacy, M., Storrie-Lombardi, L. J., et al. 2006, ApJS, 166,470

Riffel, R., Rodríguez-Ardila, A., Aleman, I., et al. 2013, MNRAS, 430, 2002

Riffel, R., Rodríguez-Ardila, A., \& Pastoriza, M. G. 2006, A\&A, 457, 61

Astropy Collaboration, Robitaille, T. P., Tollerud, E. J., et al. 2013, A\&A, 558, A33

Rodriguez, C., Taylor, G. B., Zavala, R. T., et al. 2006, ApJ, 646, 49

Rodríguez-Ardila, A., Riffel, R., \& Pastoriza, M. G. 2005, MNRAS, 364, 1041

Roebuck, E., Sajina, A., Hayward, C. C., et al. 2016, ApJ, 833, 60

Sanders, D. B., \& Mirabel, I. F. 1996, ARA\&A, 34, 749

Satyapal, S., Ellison, S. L., McAlpine, W., et al. 2014, MNRAS, 441, 1297

Satyapal, S., Secrest, N. J., Rothberg, B., et al. 2016, ApJ, 827, 58

Schawinski, K., Treister, E., Urry, C. M., et al. 2011, ApJL, 727, L31

Secrest, N. J., Dudik, R. P., Dorland, B. N., et al. 2015a, ApJS, 221, 12

Secrest, N. J., Satyapal, S., Gliozzi, M., et al. 2015b, ApJ, 798, 38

Secrest, N. J., Schmitt, H. R., Blecha, L., Rothberg, B., \& Fischer, J. 2017, ApJ, 836, 183

Seifert, W., Ageorges, N., Lehmitz, M., et al. 2010, Proc. SPIE, 7735, 7

Seifert, W., Appenzeller, I., Baumeister, H., et al. 2003, Proc. SPIE, 4841, 962

Shannon, R. M., Ravi, V., Lentati, L. T., et al. 2015, Sci, 349, 1522

Shen, Y., Liu, X., Greene, J. E., \& Strauss, M. A. 2011, ApJ, 735, 48

Smith, K. L., Koss, M., \& Mushotzky, R. F. 2014, ApJ, 794, 112
Smith, K. L., Shields, G. A., Bonning, E. W., et al. 2010, ApJ, 716, 866

Smith, K. L., Shields, G. A., Salviander, S., Stevens, A. C., \& Rosario, D. J. 2012, ApJ, 752, 63

Snyder, G. F., Hayward, C. C., Sajina, A., et al. 2013, ApJ, 768, 168

Soria, R., Hau, G. K. T., \& Pakull, M. W. 2013, ApJL, 768, L22

Springel, V. 2005, MNRAS, 364, 1105

Springel, V., \& Hernquist, L. 2003, MNRAS, 339, 289

Stern, D., Assef, R. J., Benford, D. J., et al. 2012, ApJ, 753, 30

Sutton, A. D., Roberts, T. P., Walton, D. J., Gladstone, J. C., \& Scott, A. E. 2012, MNRAS, 423, 1154

Taylor, M. B. 2005, adass XIV, 347, 29

Teng, S. H., Schawinski, K., Urry, C. M., et al. 2012, ApJ, 753, 165

Treister, E., Schawinski, K., Urry, C. M., \& Simmons, B. D. 2012, ApJL, 758, L39

Ueda, Y., Eguchi, S., Terashima, Y., et al. 2007, ApJL, 664, L79

Vacca, W. D., Cushing, M. C., \& Rayner, J. T. 2003, PASP, 115, 389

Van Wassenhove, S., Volonteri, M., Mayer, L., et al. 2012, ApJL, 748, L7

Verbiest, J. P. W., Lentati, L., Hobbs, G., et al. 2016, MNRAS, 458, 1267

Villforth, C., Hamann, F., Rosario, D. J., et al. 2014, MNRAS, 439, 3342

Villforth, C., Hamilton, T., Pawlik, M. M., et al. 2016, arXiv:1611.06236

Walton, D. J., Roberts, T. P., Mateos, S., \& Heard, V. 2011, MNRAS, 416, 1844

Wang, J.-M., Chen, Y.-M., Hu, C., et al. 2009, ApJL, 705, L76

Weisskopf, M. C., Wu, K., Trimble, V., et al. 2007, ApJ, 657, 1026

Willingale, R., Starling, R. L. C., Beardmore, A. P., Tanvir, N. R., \& O'Brien, P. T. 2013, MNRAS, 431, 394

Woo, J.-H., Cho, H., Husemann, B., et al. 2014, MNRAS, 437, 32

Wright, E. L. 2006, PASP, 118, 1711

Wright, E. L., Eisenhardt, P. R. M., Mainzer, A. K., et al. 2010, AJ, 140, 1868

Xue, Y. Q., Luo, B., Brandt, W. N., et al. 2011, ApJS, 195, 10

Xue, Y. Q., Luo, B., Brandt, W. N., et al. 2016, ApJS, 224, 15

Yan, L., Donoso, E., Tsai, C.-W., et al. 2013, AJ, 145, 55

Zolotukhin, I., Webb, N. A., Godet, O., Bachetti, M., \& Barret, D. 2016, ApJ, 817,88 